\title{
Dynamic Logit with Choice Aversion
}

\section{Citation}

Fudenberg, Drew, and Tomasz Strzalecki. 2015. "Dynamic Logit with Choice Aversion."

Econometrica 83 (2): 651-691. doi:10.3982/ecta11846. http://dx.doi.org/10.3982/ECTA11846.

\section{Published Version}

doi:10.3982/ECTA11846

\section{Permanent link}

http://nrs.harvard.edu/urn-3:HUL.InstRepos:14397609

\section{Terms of Use}

This article was downloaded from Harvard University's DASH repository, and is made available under the terms and conditions applicable to Other Posted Material, as set forth at http:// nrs.harvard.edu/urn-3:HUL.InstRepos:dash.current.terms-of-use\#LAA

\section{Share Your Story}

The Harvard community has made this article openly available.

Please share how this access benefits you. Submit a story.

\section{Accessibility}




\section{ECONOMETRICA}

JOURNAL OF THE ECONOMETRIC SOCIETY

An International Society for the Advancement of Economic Theory in its Relation to Statistics and Mathematics

http://www.econometricsociety.org/

Econometrica, Vol. 83, No. 2 (March, 2015), 651-691

DYNAMIC LOGIT WITH CHOICE AVERSION

DREW FUDENBERG

Harvard University, Cambridge, MA 02138, U.S.A.

TOMASZ STRZALECKI

Harvard University, Cambridge, MA 02138, U.S.A.

The copyright to this Article is held by the Econometric Society. It may be downloaded, printed and reproduced only for educational or research purposes, including use in course packs. No downloading or copying may be done for any commercial purpose without the explicit permission of the Econometric Society. For such commercial purposes contact the Office of the Econometric Society (contact information may be found at the website http://www.econometricsociety.org or in the back cover of Econometrica). This statement must be included on all copies of this Article that are made available electronically or in any other format. 


\title{
DYNAMIC LOGIT WITH CHOICE AVERSION
}

\author{
BY DREW FudENBERG AND TOMASZ STRZALECKI ${ }^{1}$
}

\begin{abstract}
We characterize a generalization of discounted logistic choice that incorporates a parameter to capture different views the agent might have about the costs and benefits of larger choice sets. The discounted logit model used in the empirical literature is the special case that displays a "preference for flexibility" in the sense that the agent always prefers to add additional items to a menu. Other cases display varying levels of "choice aversion," where the agent prefers to remove items from a menu if their ex ante value is below a threshold. We show that higher choice aversion, as measured by dislike of bigger menus, also corresponds to an increased preference for putting off decisions as late as possible.
\end{abstract}

KEYWORDS: Recursive choice, stochastic choice, preference for flexibility, preference for delay.

\section{INTRODUCTION}

OBSERVED INDIVIDUAL CHOICE IS TYPICALLY STOCHASTIC. Most of the theoretical literature on stochastic choice has focused on static models. Structural econometric models of aggregate dynamic decisions start by specifying a dynamic choice problem for each agent, where the agent is typically assumed to be dynamically sophisticated in the sense of correctly anticipating the way the probability distributions over his future actions depend on his actions today (Rust (1987)).

This paper provides the first axiomatic characterization of stochastic choice in dynamic settings, where choices made today can influence the possible choices available tomorrow, and consumption may (but need not) occur in multiple periods. Our main goal is to better understand the issues involved in modeling an agent who makes random choices not only over actions with immediate consumption consequences, but also over actions that can alter the choice sets that will be available in the future, when the agent's choice in each period is made taking her future randomizations into account.

Data on dynamic choice let us distinguish between models of random choice that coincide in static settings, because these models have different implications for how the agent views his future randomizations over menus, and thus

\footnotetext{
${ }^{1}$ A previous version of this paper was titled "Recursive Stochastic Choice." We thank Steve Berry, Eddie Dekel, Glenn Ellison, Phil Haile, Igal Hendel, Paulo Natenzon, Ariel Pakes, Wolfgang Pesendofer, Phil Reny, Satoru Takahashi, Jan Werner, and a co-editor and four anonymous referees for helpful comments and conversations, and Mira Frick, Jonathan Libgober, and Morgan McClellon for expert research assistance. Parts of this research were conducted while D. Fudenberg was visiting the Cowles Foundation at Yale, and T. Strzalecki was visiting the Institute for Advanced Studies at Hebrew University and then the Cowles Foundation. We are grateful for their support and hospitality, and also that of the Sloan Foundation and NSF Grants SES0954162, SES-1123729, and CAREER Grant SES-1255062.
} 
induce different choices between menus. In particular, standard models of dynamic random utility such as discounted logit generate a positive option value for each item that is added to a menu because each added item provides another chance for a good realization of the random shocks and has no downside. On the other hand, models of limited attention, implementation errors, or costly decision making all suggest that the agent may dislike adding at least some items. This leads us to propose a generalization of discounted logit called Discounted Adjusted Logit (DAL), where the attractiveness of menus is adjusted to reflect the agent's "choice aversion" and reduce or eliminate the option value of additional items.

To make this first step in characterizing dynamic stochastic choice, we maintain the Independence of Irrelevant Alternatives (IIA) assumption throughout the paper, so that static choice in our model is logit, and can be represented by a random utility model where the payoff shocks are independent and identically distributed (i.i.d.) with extreme-value type-1 distributions; see, for example, Anderson, De Palma, and Thisse (1992). Although this assumption is restrictive, we maintain it here. This lets us focus on the new issues that arise when modeling stochastic choice in a dynamic setting, axiomatize the widely used discounted logit model, ${ }^{2}$ and propose and characterize the generalization to $D A L$.

Concretely, in DAL the agent values combinations of current outcome $z_{t}$ and continuation menu $A_{t+1}$ as

$$
U_{t}\left(z_{t}, A_{t+1}\right)=v\left(z_{t}\right)+\delta \mathbb{E}\left[\max _{a_{t+1} \in A_{t+1}} U_{t+1}\left(a_{t+1}\right)+\varepsilon_{a_{t+1}}-\kappa \log \left|A_{t+1}\right|\right],
$$

and then chooses each $a_{t}=\left(z_{t}, A_{t+1}\right)$ from the menu $A_{t}$ :

$$
P_{t}\left[a_{t} \mid A_{t}\right]=\operatorname{Prob}\left[U_{t}\left(a_{t}\right)+\varepsilon_{a_{t}} \geq \max _{b_{t} \in A_{t}} U_{t}\left(b_{t}\right)+\varepsilon_{b_{t}}\right],
$$

where $\varepsilon_{a_{t}}$ has an extreme-value distribution.

Every DAL is equivalent to logit in static decisions or when $\delta=0$, regardless of the value of $\kappa$. DAL with $\kappa=0$ reduces to the usual form of discounted logit. The new parameter $\kappa$ measures the agent's choice aversion, and is determined by the extent to which the agent wants to add new items to the menu. When $\kappa=0$, the agent is choice loving in the sense that the agent always prefers to add additional items to a menu, as in the "preference for flexibility" of Kreps (1979) and Dekel, Lipman, and Rustichini (2001).

Another special case of interest is $\kappa=1$; here, the agent wants to remove choices that are worse than average, as might be the case if the agent were

\footnotetext{
${ }^{2}$ Miller (1984), Rust (1989), Hendel and Nevo (2006), Kennan and Walker (2011), Sweeting (2011), Gowrisankaran and Rysman (2012), and surveys Eckstein and Wolpin (1989), Rust (1994), Aguirregabiria and Mira (2010).
} 
worried about choosing them by accident or if the agent incurs "menu" or "consideration" costs that exactly offset the benefit of another random draw. ${ }^{3}$

In addition to DAL, we present two alternative but equivalent representations. To motivate the representations and explain their equivalence, it is useful to recall that there are several explanations in the literature for stochastic choice in static problems.

Random utility: Agents might maximize their expected utility given privately observed payoff shocks, as in Thurstone (1927), Marschak (1959), McFadden (1973), and Harsanyi (1973a), so that even choices that are typically unappealing could be optimal when the payoff shock is large. Logit corresponds to payoff shocks that are i.i.d. with extreme-value type-1 distributions; this functional form is the starting point for the discounted logit model.

Inattention: Agents might randomize as the result of error or inattention. van Damme (1991) and van Damme and Weibull (2002) studied a model where the agent's intended choice is implemented with a probability that depends on the attention paid, and Manzini and Mariotti (2014) axiomatized a model where each possible choice is overlooked with an exogenous probability. Static logit corresponds to the case where the agent chooses a probability distribution to maximize the difference between the expected utility and the relative entropy of the choice distribution with respect to the uniform distribution, as in Mattsson and Weibull (2002).

In our dynamic setting, the relative entropy cost function corresponds to $\kappa=1$ in an alternative representation we call Discounted Adjusted Entropy or DAE. In this case, adding an equally good item to a singleton menu has no effect on the menu's value, as the agent will choose to randomize uniformly and thus incur no attention cost while receiving an equivalent current outcome. As with DAL, all versions of DAE are equivalent to logit in static decisions, but the value of $\kappa$ matters once there are two or more periods. In particular, DAE with $\kappa=0$, where the cost function is the negative of the entropy of the choice probabilities, is equivalent to discounted logit and so is choice loving; here, the preference for larger choice sets arises from the fact that larger choice sets have a higher maximum entropy.

Ambiguity aversion: Agents might be uncertain about the rewards to each choice, and randomize in response to ambiguity aversion. As shown by Fudenberg, Iijima, and Strzalecki (2014), this can lead to the agents acting as if their goal was to maximize the sum of expected utility and entropy (or some other convex function); thus, this explanation for stochastic choice and the explanation based on inattention motivate representations with identical functional forms, including DAE as a the special case where IIA is satisfied. ${ }^{4}$

\footnotetext{
${ }^{3}$ When $\kappa \in(0,1)$, the agent prefers to include additional items provided they are not too much worse than the current average, while when $\kappa>1$, the agent only wants to add items that are sufficiently better.

${ }^{4}$ Harsanyi (1973b) introduced the more general idea that choice probabilities correspond to maximizing the sum of expected utility and a nonlinear perturbation function. This functional
} 
Weighting function: Finally, observed choices might be the result of psychophysical "weighting functions," as in Luce (1959), who characterized stochastic choice in static problems under the additional assumptions of positivity (all actions have positive probability) and IIA. Under these assumptions, the observed choice distribution can be generated by assigning weights $W(a)$ to each action $a$, and then picking an action with probability equal to its share of the total weight; the term "psychophysical" reflects the idea that these weights correspond to the mental stimuli generated by the feasible actions. This motivation for random choice corresponds to our Discounted Adjusted Luce (DALu) representation.

As should be clear by now, this paper relates to several strands of the axiomatic decision theory literature, to foundational literature in game theory, and to empirical work on dynamic choice. We discuss these relationships in the concluding section, after we have developed our representations and the associated axioms.

Of these axioms, the most significant are: IIA (so that static choice is logit); a separability axiom that implies that preferences over future decision problems are independent of the outcome in the current period, and vice versa; a recursivity axiom that links current choice of menus for tomorrow to behavior when tomorrow arrives; and a replica invariance axiom that says roughly that how the agent feels about duplicating every item in a menu does not depend on the menu's elements or size. We also characterize the special case of $\kappa=0$ (which corresponds to the usual discounted logit) with a more specific "aggregate recursivity" axiom, which says that menu $A$ is more likely to be selected now than some other menu $B$ if elements of $A$ are more likely to be selected than elements of $B$ when both are presented as an immediate decision next period. In contrast, DAE with $\kappa=1$ corresponds to "average recursivity," where menu $A$ is preferred if its elements are chosen with higher average probability. As in the deterministic case of Koopmans (1960), additional assumptions are needed to arrive at a stationary discounted sum formulation.

In addition to its impact on preferences over menus at a given time, the choice aversion parameter $\kappa$ also influences whether the agent wants to make decisions as soon as possible or to postpone them: when $\kappa=0$, the agent enjoys making decisions and so (because of discounting) prefers to decide early, while when $\kappa \geq 1$, the agent views decisions as costly and wishes to postpone them. As we argue below, to the extent that people typically do prefer to postpone decisions, this can be viewed as an additional reason to generalize DAL to allow for positive values of $\kappa$.

form was recently used by Swait and Marley (2013) to model stochastic choice as the result of balancing multiple goals. 


\section{DYNAMIC CHOICE PROBLEMS}

For any set $S$, let $K(S)$ be the collection of nonempty finite subsets of $S$, to be interpreted as the collection of possible choice problems. For any set $S$ let $\Delta(S)$ be the collection of probability measures on $S$ with finite support. Let $\Delta^{n}:=\Delta(\{1, \ldots, n\})$.

We assume that time is discrete, $t=0,1, \ldots, T$ with $T$ finite. We assume throughout that $T>0$, that is, choices are observed in at least two time periods. Let $Z$ be the set of all one-period outcomes. ${ }^{5}$ In any period $t$, an individual choice problem is called a menu; we denote period- $t$ menus by letters $A_{t}, B_{t}, C_{t}, \ldots$ and the space in which all menus live by $\mathcal{M}_{t}$. The elements of the menu are called actions and are denoted by $a_{t}, b_{t}, c_{t}, \ldots$; the space in which all actions live is denoted by $\mathcal{A}_{t}$. We construct the set of dynamic choice problems recursively. Let $\mathcal{A}_{T}:=Z$ and $\mathcal{M}_{T}:=K\left(\mathcal{A}_{T}\right)$; in period $T$, actions are synonymous with one-period outcomes because in the terminal period there is no future, and period- $T$ menus are just collections of one-period outcomes. Now we define the possible menus and actions in earlier time periods by $\mathcal{A}_{t}:=Z \times \mathcal{M}_{t+1}$ and $\mathcal{M}_{t}:=K\left(\mathcal{A}_{t}\right)$. Thus, an action $a_{t}$ at time $t$ is a pair $\left(z_{t}, A_{t+1}\right)$ of a current outcome and a time- $t+1$ menu, while a menu $A_{t}$ at time $t$ is a finite set of such actions. For notational convenience, we set $\mathcal{M}_{T+1}=\emptyset$ and use the convention that $Z \times \mathcal{M}_{T+1}=Z$.

It is important that the actions today can restrict future opportunities without having any impact on the current outcome; for example, the agent might face the period- $T-1$ menu $\left\{\left(z, A_{T}\right),\left(z, A_{T}^{\prime}\right)\right\}$. Moreover, the agent might face the choice at time $T-3$ of whether to commit to her time- $T$ outcome in period $T-2$ or in period $T-1$. As we will see, different values of $\kappa$ predict different choices here; this is one advantage of allowing a general finite horizon as opposed to restricting the model to have only two time periods.

Definition 1-Dynamic Stochastic Choice Rule: A dynamic stochastic choice rule is a collection of mappings $P=\left\{P_{t}\right\}_{t=0}^{T}$ such that $P_{t}: \mathcal{M}_{t} \rightarrow \Delta\left(\mathcal{A}_{t}\right)$, with the property that, for any $A_{t} \in \mathcal{M}_{t}$, the support of $P_{t}\left(A_{t}\right)$ is a subset of $A_{t}{ }^{6}$

For any $A_{t} \in \mathcal{M}_{t}, P_{t}\left(A_{t}\right)$ is the probability distribution on actions that represents the stochastic choice from $A_{t}{ }^{7}$ For notational convenience, we write

\footnotetext{
${ }^{5}$ Our richness axiom will imply that $Z$ is infinite, but we do not assume any structure on this set; possible cases include: a subset of $\mathbb{R}$ (monetary payoffs), or $\mathbb{R}^{n}$ (consumption bundles or acts), and $\Delta\left(\mathbb{R}^{n}\right)$ (lotteries).

${ }^{6}$ This is the stochastic version of the usual condition that the only elements that can be chosen are those that belong to the menu.

${ }^{7}$ Note that this implicitly assumes that choice at time $t$ from a given menu is independent of past history, although that history can influence the set of options available. We believe that our approach could be extended to allow for history dependence through a state variable, as is com-
} 
$P_{t}\left[B_{t} \mid A_{t}\right]$ to denote the probability that the chosen action will belong to the set $B_{t}$ when the choice set is $A_{t}$. For $\left(z, A_{t+1}\right) \in A_{t}$, we write $P_{t}\left[\left(z, A_{t+1}\right) \mid A_{t}\right]$ instead of $P_{t}\left[\left\{\left(z, A_{t+1}\right)\right\} \mid A_{t}\right]$; note that $P_{t}\left[B_{t} \mid A_{t}\right]=\sum_{b_{t} \in B_{t}} P_{t}\left[b_{t} \mid A_{t}\right]$.

Note that the time periods in our model are data that are observed by the analyst, as opposed to the purely notional time periods in nested logit, which is an as-if representation of static choice. As we will see, observing how the choice probabilities vary with the times that decisions are made provides additional information about the agent's preferences that is unavailable in static models.

Our primitive is a dynamic stochastic choice rule $P$. However, it will be more convenient to express some of our questions using the notion of the stochastic preference $\succsim_{t}$ on $A_{t}$, which is derived from $P_{t}$ as follows.

DEFINITION 2-Stochastic Preference: Action $a_{t}$ is stochastically preferred to action $b_{t}$ at time $t$, denoted $a_{t} \succsim_{t} b_{t}$, if $P_{t}\left[a_{t} \mid\left\{a_{t}, b_{t}\right\}\right] \geq P_{t}\left[b_{t} \mid\left\{a_{t}, b_{t}\right\}\right]$. Given $\succsim_{t}$, we define induced stochastic preferences on $Z$ and $\mathcal{M}_{t+1}$ as follows: $z$ is stochastically preferred to $w$ at time $t$, denoted $z \succsim_{t} w$, if $\left(z, A_{t+1}\right) \succsim_{t}\left(w, A_{t+1}\right)$ for all $A_{t+1} \in \mathcal{M}_{t+1}$. Menu $A_{t+1}$ is stochastically preferred to $B_{t+1}$ at time $t$, denoted $A_{t+1} \succsim_{t} B_{t+1}$, if $\left(z, A_{t+1}\right) \succsim_{t}\left(z, B_{t+1}\right)$ for all $z \in Z$.

Under our basic axioms (to be introduced later), these stochastic preference relations are complete and transitive. All behavioral content expressed in terms of $\succsim_{t}$ can be formulated in terms of $P_{t}$ at the cost of making some expressions more cumbersome.

\section{CHOICE RULES AND THEIR REPRESENTATIONS}

We study three equivalent representations of a dynamic stochastic choice rule. Each of these writes values as the sum of current payoff plus the discounted value of the expected continuation payoff, where the continuation payoff assigned to each menu is adjusted to account for its size.

The most familiar-looking of the representations is Discounted Adjusted Logit, which generalizes the standard discounted logit representation used to model dynamic individual choice in estimation problems.

DEFINITION 3: A random variable $\varepsilon$ has the extreme value distribution (with noise parameter 1), denoted $\varepsilon \sim \mathrm{EV}(1)$, if its c.d.f. is $F(\varepsilon)=$ $\exp (-\exp (-\varepsilon-\gamma))$, where $\gamma$ is Euler's constant.

DefinITION 4-Discounted Adjusted Logit: $P$ has a Discounted Adjusted Logit (DAL) representation iff there exist a utility function $v: Z \rightarrow \mathbb{R}$, dis-

monly allowed in empirical work (see, e.g., Aguirregabiria and Mira (2010)). However, because the complexities involved in axiomatically characterizing state dependence seem orthogonal to the study of stochastic choice, we have not tried to develop this extension. 
count factor $\delta \in(0,1)$, choice aversion parameter $\kappa \geq 0$, and value functions $U_{t}: \mathcal{A}_{t} \rightarrow \mathbb{R}$ recursively defined by $U_{T}(z)=v(z)$ and

$$
U_{t}\left(z_{t}, A_{t+1}\right)=v\left(z_{t}\right)+\delta \mathbb{E}\left[\max _{a_{t+1} \in A_{t+1}} U_{t+1}\left(a_{t+1}\right)+\varepsilon_{a_{t+1}}-\kappa \log \left|A_{t+1}\right|\right],
$$

such that, for all $t=0, \ldots, T$, all $A_{t}$, and all $a_{t} \in A_{t}$,

$$
P_{t}\left[a_{t} \mid A_{t}\right]=\operatorname{Prob}\left[U_{t}\left(a_{t}\right)+\varepsilon_{a_{t}} \geq \max _{b_{t} \in A_{t}} U_{t}\left(b_{t}\right)+\varepsilon_{b_{t}}\right],
$$

where $\varepsilon_{a_{t}} \sim^{\text {i.i.d. }} \mathrm{EV}(1){ }^{8}$

In this representation, the $\varepsilon$ terms correspond to payoff shocks that are observed by the decision maker but not by the analyst, as in static random utility models. ${ }^{9}$ Note that these payoff shocks apply to every action, just as they do under the "Assumption AS" or equation (3.7) of Rust (1994). As an example, suppose that a consumer first decides what size tuna fish can to buy and later decides how much to consume each day; then payoff shocks apply to each possible purchasing decision in period $t=0$ as well as to consumption in subsequent periods, as in Hendel and Nevo (2006, p. 1645).

We call this sort of payoff shocks "shocks to actions," as opposed to the alternative model where payoff shocks apply only to a set of "immediate outcomes." The simplest version of this alternative model, in which the agent knows all future shocks from the outset, predicts purely deterministic choice in settings such as the purchasing decisions discussed above, which seems implausible and is impractical for empirical work. ${ }^{10}$ The model could generate stochastic decisions under the assumption that the agent gradually learns about future payoff shocks over time, but this would make it difficult or impossible to obtain closed form solutions, and so require computationally intensive numerical work. Thus we follow the empirical literature here and restrict attention to "shocks to actions." We explore one consequence of this modeling assumption in Section 5.4.

In the case $\kappa=0$, the representation reduces to discounted logit. (More precisely, it is the simplest sort of discounted logit representation, as it does not include a state variable and assumes stationarity; we make these simplifications

\footnotetext{
${ }^{8}$ Allowing for a more general noise parameter $\eta$ is possible, but does not lead to a more general model, as only $v / \eta$ is identified.

${ }^{9}$ Note that the agent does not know the realizations of future payoff shocks, so he is on an equal footing with the analyst when it comes to future. This simplifying assumption makes the model tractable, which is why it is used in estimation.

${ }^{10}$ Some aspects of this alternative approach have been studied in the decision theory literature: Ahn and Sarver (2013) related deterministic choice over menus in period 0 with random choice from menus in period 1, but it is not clear how to extend this to allow for random choice in period 0. Dillenberger, Lleras, Sadowski, and Takeoka (2013) and Lu (2013) made a similar connection in a model with an objective state space, but likewise did not characterize random choice in period 0 .
} 
to focus on the issues related to recursive choice.) As we will show explicitly below, discounted logit is choice loving in the sense of always preferring larger menus. Intuitively, this comes from the fact that each new object added to the menu provides another chance for a good realization of the random shock $\varepsilon$, as it does in any random utility model unless the shocks to some of the objects are perfectly correlated. ${ }^{11}$

The case $\kappa=1$ represents error-averse choice in the sense that the constant $\log \left|A_{t+1}\right|$ that the agent prefers not to add a new item to a singleton menu if it is worse than the current item (in the sense of being chosen less than half the time in the binary menu). More generally, the parameter $\kappa$ is responsible for how heavily big sets are penalized and corresponds to the choice aversion ordering of agents introduced below. The role of $\kappa$ and the special nature of $\kappa=1$ are easier to see in the next two representations.

DEFINITION 5: For any $q \in \Delta^{n}$, let $H^{n}(q):=-\sum_{i=1}^{n} q_{i} \log \left(q_{i}\right)$ be the entropy of $q$ with the convention that $0 \log 0=0$. Let $J_{\kappa}^{n}(q)=H^{n}(q)-\kappa \log n$ be the adjusted entropy.

Since the entropy and adjusted entropy are invariant to permutations, we treat distributions $q \in \Delta(A)$ as if they were elements of $\Delta^{|A|}$ when they are arguments of the functions $H$ and $J$.

Definition 6-Discounted Adjusted Entropy: $P$ has a Discounted Adjusted Entropy (DAE) representation if and only if there exist a utility function $v: Z \rightarrow \mathbb{R}$, discount factor $\delta \in(0,1)$, choice aversion parameter $\kappa$, and value functions $U_{t}: \mathcal{A}_{t} \rightarrow \mathbb{R}$ recursively defined by $U_{T}(z)=v(z)$ and

$$
\begin{aligned}
& U_{t}\left(z_{t}, A_{t+1}\right) \\
& \quad=v\left(z_{t}\right)+\delta\left[\max _{q \in \Delta\left(A_{t+1}\right)} \sum_{a_{t+1} \in A_{t+1}} q\left(a_{t+1}\right) U_{t+1}\left(a_{t+1}\right)+J_{\kappa}^{\left|A_{t+1}\right|}(q)\right],
\end{aligned}
$$

such that, for all $t=0, \ldots, T$ and $A_{t}$,

$$
P_{t}\left[\cdot \mid A_{t}\right]=\underset{q \in \Delta\left(A_{t}\right)}{\arg \max } \sum_{a_{t} \in A_{t}} q\left(a_{t}\right) U_{t}\left(a_{t}\right)+J_{\kappa}^{\left|A_{t+1}\right|}(q) .
$$

\footnotetext{
${ }^{11}$ The standard nested logit model applies to a static choice of an item and so cannot directly address preferences over menus, but a similar issue arises there: If "purchase" is one nest and "not purchase" is another, then in the limit of a very large set of goods, almost everyone must purchase. Ackerberg and Rysman (2005) proposed (but did not characterize) two alternative responses to this issue in a static model: either scale the variance of the extreme-value shocks with the number of goods in the menu, or add a term to the utility function that depends on various characteristics of the menu. Fudenberg, Iijima, and Strzalecki (2014) axiomatized an extension of the static nested logit model that allows for choice aversion.
} 
Note that the entropy of the uniform distribution over $n$ objects is $\log (n)$, which increases without bound in $n$. Hence a $\kappa=0$ agent will prefer a menu of many roughly similar objects to the singleton menu with just one of them. We elaborate on the consequences of this below.

When $\kappa=1$, the adjusted entropy is $-R^{n}(q)$, the negative of relative entropy of $q$ with respect to the uniform distribution. This function is maximized in the interior of the simplex, so when $\kappa=1$, stochastic choice can be interpreted as the result of costly attention: Choosing a uniform distribution is costless, but departing from it in the direction of a more desirable lottery is costly, with cost proportional to the relative entropy. Such an agent is error-averse, and prefers removing the lowest-ranked item from a menu, but is indifferent about whether an equally good item is added to a singleton menu. As the equivalent logit representation suggests, though, the error-averse preferences are consistent with a combination of consideration costs based on menu size and logit-type payoff shocks.

More generally, note that $J_{\kappa}^{n}(q)=(1-\kappa) H^{n}(q)-\kappa R^{n}(q)$, where $R^{n}(q)=$ $\sum_{i=1}^{n} q_{i} \log \frac{q_{i}}{1 / n}$ is the relative entropy of $q$ with respect to the uniform distribution. That is, adjusted entropy is a linear combination of entropy and relative entropy. Thus, adjusted entropy represents a combination of two motivations for random choice: desire for randomization (represented by the entropy term) and costly attention (represented by the negative relative entropy term).

The third representation is perhaps the easiest to use in applications, as it incorporates the well-known "log-sum" representation of the logit value function. (See, e.g., Train (2009, Chapter 3), or Lemma 3 in the Appendix.)

DEFINITION 7-Discounted Adjusted Luce: $P$ has a Discounted Adjusted Luce (DALu) representation if there exist a utility function $v: Z \rightarrow \mathbb{R}$, discount factor $\delta \in(0,1)$, choice aversion parameter $\kappa \in \mathbb{R}$, and value functions $W_{t}: \mathcal{A}_{t} \rightarrow \mathbb{R}_{++}$recursively defined by $\log W_{T}(z)=v(z)$ and

$$
\log W_{t}\left(z_{t}, A_{t+1}\right)=v\left(z_{t}\right)+\delta\left[\log \left(\sum_{a_{t+1} \in A_{t+1}} W_{t+1}\left(a_{t+1}\right)\right)-\kappa \log \left|A_{t+1}\right|\right],
$$

such that, for all $A_{t}$ and all $a_{t} \in A_{t}$,

$$
P_{t}\left[a_{t} \mid A_{t}\right]=\frac{W_{t}\left(a_{t}\right)}{\sum_{b_{t} \in A_{t}} W_{t}\left(b_{t}\right)} .
$$

Here, as in the static Luce case, the ratio of the choice probabilities of two items is given by the ratio of their weights; these weights now correspond to a weighted sum of current payoff and the sum of the weights of the continuation menus, with menu size penalized by $\kappa$.

The following proposition states the equivalence of the three representations. 
PROPOSITION 1: The following statements are equivalent:

1. $P$ has a DAL representation with parameters $(v, \delta, \kappa)$ and value functions $U_{t}$.

2. $P$ has a $D A E$ representation with parameters $(v, \delta, \kappa)$ and value functions $U_{t}$.

3. $P$ has a DALu representation with parameters $(v, \delta, \kappa)$ and value functions $W_{t}=\exp \left(U_{t}\right)$.

Moreover, if $P$ has any of the above representations with parameters $(v, \delta, \kappa)$ and $\left(v^{\prime}, \delta^{\prime}, \kappa^{\prime}\right)$, then $(v, \delta, \kappa)=\left(v^{\prime}+\beta, \delta^{\prime}, \kappa^{\prime}\right)$ for some constant $\beta$.

The proof is in the Appendix (as are the proofs of all of the other propositions). In outline, the proof first notes that all three representations generate the same choice probabilities as logit in the static case and thus are equivalent in final period, and then uses the equivalence of the corresponding value functions for final-period menus to work backwards. The reason that $\alpha v$ is not equivalent to $v$ in DAL, as opposed to the usual affine uniqueness of expected utility, is that we set the parameter of the extreme-value distribution to 1, which fixes the multiplicative term in the utility function; this parallels our specification of a unit coefficient for adjusted entropy in DAE and of $W=\exp (1 \cdot v)$ in DALu. Uniqueness of $\delta$ is as in the deterministic choice literature, and uniqueness of $\kappa$ follows from the period- $t$ choices between singleton menus at period $t+1$ and arbitrary two-element menus. Motivated by this proposition, we refer to the three representations collectively as discounted adjusted representations, or DARs.

DEFINITION 8-Discounted Adjusted Representation: $P$ has a DAR if it has a DAL, DAE, or a DALu representation.

\section{APPLICATIONS}

\subsection{Work or College?}

To illustrate our setup, consider the following example of a high school student's choice of whether or not to go to college, which we adapt from Train (2009, Chapter 7). There are two periods. In period 0, the student can either go to college, which leads to immediate outcome $c$, or work, which leads to immediate outcome $w$. In addition to immediate outcomes, her choices in period 0 have consequences for the sets of options available in period 1: If the student works in period 0 , she must work in job $z$ in period 1 , and if the student goes to college in period 0 , she will choose between two jobs $x$ and $y$. Thus, the student faces the decision depicted in Figure 1.

To represent this decision tree as one of our dynamic choice problems, let $A_{1}=\{x, y\}$ and $B_{1}=\{z\}$ be the two possible continuation problems in period 1 (after choosing to go to college or not). Then the time zero choice problem is $A_{0}=\left\{\left(c, A_{1}\right),\left(w, B_{1}\right)\right\}$. We write $P_{0}\left[\left(c, A_{1}\right) \mid A_{0}\right]$ to denote the probability that 


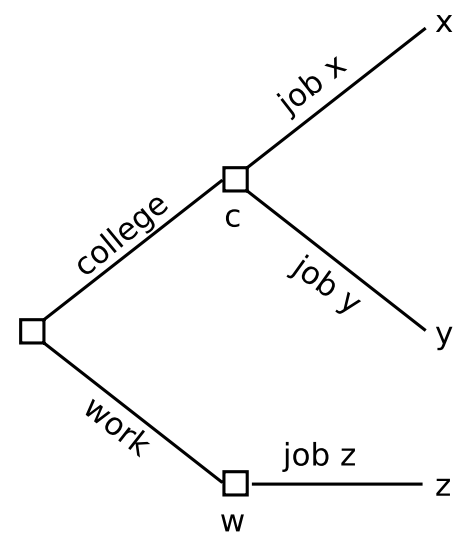

FIGURE 1.-Choosing whether to go to college.

the student chooses to go to college in period 0 and $P_{1}\left[x \mid A_{1}\right]$ to denote the probability that in period 1 (conditional on having gone to college) the student chooses a job $x$.

Suppose that the $v$ is the utility function of the agent, so that, for example, the utility of outcome $c$ is $v(c)$. Under any of the DARs, the value of the continuation choice problem $A_{1}$ is $\log \left(e^{v(x)}+e^{v(y)}\right)-\kappa \log 2$ and the probability of choosing job $x$ from $A_{1}$ is $\frac{e^{v(x)}}{e^{v(x)}+e^{v(y)}}$. Using the formula from DALu, it is immediate that the probability that the student goes to college is

$$
\begin{aligned}
P_{0}\left[\left(c, A_{1}\right) \mid A_{0}\right]= & \exp \left(v(c)+\delta \log \left(e^{v(x)}+e^{v(y)}\right)-\delta \kappa \log 2\right) \\
& /\left(\exp \left(v(c)+\delta \log \left(e^{v(x)}+e^{v(y)}\right)-\delta \kappa \log 2\right)\right. \\
& +\exp (v(w)+\delta v(z))) .
\end{aligned}
$$

An extreme case is when $v(x)=v(y)=v(z)=v_{1}$ and $v(c)=v(w)=v_{0}$. Then

$$
P_{0}\left[\left(c, A_{1}\right) \mid A_{0}\right]=\frac{e^{v_{0}+\delta\left(v_{1}+(1-\kappa) \log 2\right)}}{e^{v_{0}+\delta\left(v_{1}+(1-\kappa) \log 2\right)}+e^{v_{0}+\delta v_{1}}} .
$$

Here the $\log 2$ term reflects the fact that going to college leads to two future options. When $\kappa<1$, the term has positive weight, corresponding to an agent who likes to have choices, perhaps due to the perceived option value; when $\kappa>1$, the $\log 2$ term has negative weight, corresponding to an agent who is choice-averse. When $\kappa=1$, the $\log 2$ term vanishes, so the agent is indifferent about adding an equally good choice to the menu. 


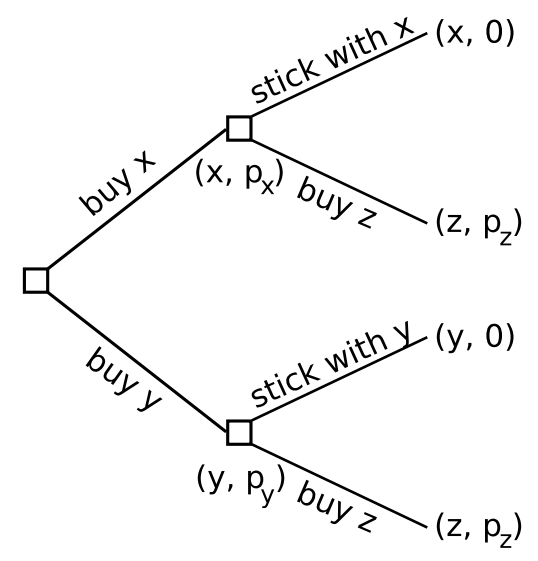

FIGURE 2.-Durable goods purchasing decisions.

\subsection{Durable Goods}

The example in Figure 2 shows how the model of individual demand for durable goods proposed by Gowrisankaran and Rysman (2012) fits into our framework. In period $t=0$, there are two durable goods available: $x$ and $y$; in period $t=1$, there is only one durable good $z$ available. In this environment, the customer also cares about the price of each good, so in our setting, the outcomes available in each period are pairs (good, price), for example, $\left(x, p_{x}\right) .^{12}$

In period $t=0$, the agent chooses between $x$ and $y$. She will receive the flow of utility of that choice in $t=0$ as well as in $t=1$, unless she decides to replace it with $z$. For example, purchasing $x$ at price $p_{x}$ corresponds to the action $a=\left(\left(x, p_{x}\right), A_{1}\right)$ : the agent receives $x$ in period 0 and pays the price $p_{x}$; in period 1 , the agent has a choice from the menu $A_{1}=\left\{(x, 0),\left(z, p_{z}\right)\right\}$ : not making any purchases and continuing to receive $x$, or making a new purchase $z$ and paying the price $p_{z}$. Likewise, purchasing $y$ at price $p_{y}$ corresponds to the action $b=\left(\left(y, p_{y}\right), B_{1}\right)$, where $B_{1}=\left\{(y, 0),\left(z, p_{z}\right)\right\}$.

Like us, Gowrisankaran and Rysman (2012) supposed that the individual is uncertain about her own future choices, and moreover, that this uncertainty is symmetric between the agent and the analyst. In addition, they made the same functional form assumptions that we do: They assumed that in period 1, the utility of the outcome $(x, 0)$ is $v(x)+\varepsilon_{x, 1}$, the utility of $(y, 0)$ is $v(y)+\varepsilon_{y, 1}$, and the utility of $(z, r)$ is $v(z)-r+\varepsilon_{z, 1}$, where $\varepsilon_{x, 1}, \varepsilon_{y, 1}, \varepsilon_{z, 1} \sim$ i.i.d. $\operatorname{EV}(1)$. Gowrisankaran and Rysman (2012) assumed that preferences are discounted

\footnotetext{
${ }^{12}$ Gowrisankaran and Rysman (2012) also allowed for the possibility that the agent may not know the set of goods available in future periods, nor their prices. With incomplete information, their model is not formally a special case of ours. We abstract from this and assume complete information on the part of the agent.
} 
logit; in particular, they assumed that the taste shocks in period 0 and period 1 are independent and that the period- 1 shock is not revealed until the beginning of period 1 . Thus their model corresponds to DAR with $\kappa=0$, so from the agent's $t=0$ perspective, the value of menu $A_{1}$ is

$$
V\left(A_{1}\right)=\log \left(e^{u(x)}+e^{u(z)-p_{z}}\right) .
$$

Likewise,

$$
V\left(B_{1}\right)=\log \left(e^{u(y)}+e^{u(z)-p_{z}}\right) .
$$

As a result, the probability that the agent purchases $x$ in period 0 is

$$
\frac{e^{u(x)-p_{x}+\delta V\left(A_{1}\right)}}{e^{u(x)-p_{x}+\delta V\left(A_{1}\right)}+e^{u(y)-p_{y}+\delta V\left(B_{1}\right)}} .
$$

\subsection{Magazine Subscriptions}

Our framework can be also used to model choices that influence the future opportunities to make decisions, such as choosing between liquid and illiquid assets, purchasing or renting a car, or whether to get a cellphone with or without a contract. For example, suppose that an agent is contemplating buying a magazine subscription; if the agent does not buy the subscription, she will have to decide whether to purchase each issue separately. For simplicity, we abstract from pricing (since subscriptions offer cost savings, this force would push the agent in the direction of buying the subscription). We focus instead on another aspect of the problem: since every issue differs, even if in expectation the magazine is worth buying, there may be weeks when it is not. Thus, not having a subscription offers an option value. On the other hand, the agent may be choice-averse and may prefer to make the decision once and for all, instead of having to decide every week. As we will see, in our model the strength of this force is measured by the parameter $\kappa$.

To illustrate this in a simple model, suppose that in period $t=0$, the agent makes the subscription decision, and in periods $t=1,2$, she makes decisions about buying individual issues should she choose not to subscribe. Let $x$ denote 'consuming' the magazine and $\diamond$ denote not consuming it. The agent faces the dynamic decision problem depicted in Figure 3.

Formally, buying a subscription corresponds to the action $b_{0}=(\diamond,\{(x,\{x\})\})$, whereas not buying the subscription corresponds to $n_{0}=(\diamond,\{(x,\{x, \diamond\})$, $(\diamond,\{x, \diamond\})\})$. Normalize $v(x)=0$ and let $v(\diamond)=d<0$. Then the probability of skipping the purchase of an issue of the magazine is the same in every period and equals

$$
\pi:=P_{1}[(\diamond, A) \mid\{(\diamond, A),(x, A)\}]=P_{2}[\diamond \mid\{\diamond, x\}]=\frac{e^{d}}{1+e^{d}},
$$




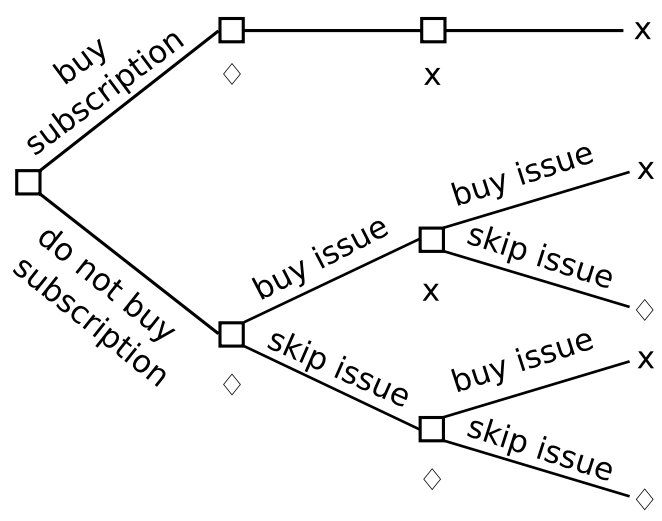

FIGURE 3.-Subscription decision.

which is increasing in $d$. With this parameterization, buying the subscription has value $U_{0}\left(b_{0}\right)=d$, whereas not buying the subscription has value

$$
\begin{aligned}
U_{0}\left(n_{0}\right) & =d+\delta\left(\log \left(e^{d+\delta(\log (1+e)-\kappa \log 2)}+e^{\delta(\log (1+e)-\kappa \log 2)}\right)-\kappa \log 2\right) \\
& =d+\left(\delta+\delta^{2}\right) \log \left(\frac{1+e^{d}}{2^{\kappa}}\right) .
\end{aligned}
$$

Thus, the agent will buy the subscription with probability more than 0.5 iff her choice aversion parameter $\kappa$ is above the threshold

$$
\kappa^{*}=\frac{\log \left(1+e^{d}\right)}{\log 2}=-\frac{\log (1-\pi)}{\log 2} .
$$

Note that $\kappa^{*}$ is increasing in $\pi$, which can thus be seen as a measure of the option value of not locking in to the subscription: the higher the probability of skipping the single issue purchase, the stronger the choice aversion has to be to compensate for the forgone flexibility.

As we show in Section 5, it is true more generally that the parameter $\kappa$ measures how the agent trades off flexibility and decision costs. The ability to quantify this tradeoff may be helpful in applied work studying choices about subscriptions and other choices influencing the future opportunities to make decisions, such as those mentioned in Section 4.

\section{COMPARATIVE STATICS}

\subsection{Choice Aversion}

We now introduce a partial order that allows us to compare how agents feel about adding items to a menu. 
DEFINITION 9-Choice Aversion: Agent 2 is more choice-averse than agent 1 if, for all menus $A_{t+1}, B_{t+1}$ with $\left|A_{t+1}\right| \leq\left|B_{t+1}\right|, A_{t+1} \succsim_{t}^{1} B_{t+1}$ implies that $A_{t+1} \succsim_{t}^{2} B_{t+1}$ and $A_{t+1} \succ_{t}^{1} B_{t+1}$ implies that $\bar{A}_{t+1} \succ_{t}^{2} B_{t+1}$.

Proposition 2: Consider any two DARs $P$ with parameters $(v, \delta, \kappa)$, and $P^{*}$ with parameters $\left(v, \delta, \kappa^{*}\right)$. Then, $P$ is more choice-averse than $P^{*}$ if $\kappa \geq \kappa^{*}$. The converse is true under the Richness axiom (Axiom 5) introduced in Section 6.

Intuitively, this is true because higher $\kappa$ means that bigger sets are penalized more heavily. The parameter $\kappa$ also determines the conditions under which the agent wants to add an item to a menu, that is, when $A_{t+1} \cup\left\{b_{t+1}\right\} \succ_{t} A_{t+1}$ : the higher the $\kappa$, the more "choosy" the agent.

PROPOSITION 3: For any DAR with parameter $\kappa$ and $b_{t+1} \notin A_{t+1}$, we have $A_{t+1} \cup\left\{b_{t+1}\right\} \succ_{t} A_{t+1}$ if and only if $P_{t+1}\left[b_{t+1} \mid A_{t+1} \cup\left\{b_{t+1}\right\}\right]>1-\frac{\left|A_{t+1}\right|^{\kappa}}{\left(\left|A_{t+1}\right|+1\right)^{\kappa}}$. In particular, if $A_{t+1}=\left\{a_{t+1}\right\}$, the agent strictly prefers $\left\{a_{t+1}, b_{t+1}\right\}$ to $\left\{a_{t+1}\right\}$ iff $P_{t+1}\left[b_{t+1} \mid\left\{a_{t+1}, b_{t+1}\right\}\right]>\frac{2^{\kappa}-1}{2^{\kappa}}$.

Thus if $\kappa=0$, the agent always wants to add new items. If $\kappa=1$, the agent only wants to add a new item if it is chosen with more than uniform probability, which fits with the interpretation that stochastic choice arises from a cost of preventing errors. This provides an easy way to distinguish $\kappa=0$ from $\kappa=1$ (assuming that the model is correct with one of these two values): It is sufficient to observe the agent's choice between the menu $\left\{a_{t+1}, a_{t+1}^{\prime}\right\}$ and the menu $\left\{a_{t+1}\right\}$, where $P_{t}\left[a_{t+1} \mid\left\{a_{t+1}, a_{t+1}^{\prime}\right\}\right]=0.5$. Moreover, by observing the agent's choice probabilities between $n$ pairs of menus, we can determine to which of $n+1$ subintervals the agent's value of $\kappa$ belongs. ${ }^{13}$ The next proposition provides further information on the implications of various values of $\kappa$ by linking them to stochastic versions of axioms in the literature on preferences over menus: Kreps (1979), Dekel, Lipman, and Rustichini (2001, 2009), Gul and Pesendorfer (2001).

Proposition 4: Suppose that $P$ is a DAR. Then

1. $\kappa \geq 1$ iff for all $t$ and disjoint $A_{t+1}, B_{t+1}, A_{t+1} \succsim_{t} B_{t+1}$ implies $A_{t+1} \succsim_{t} A_{t+1} \cup$ $B_{t+1}$.

2. $\kappa \leq 1$ iff for all $t$ and disjoint $A_{t+1}, B_{t+1}, A_{t+1} \succsim_{t} B_{t+1}$ implies $A_{t+1} \cup B_{t+1} \succsim_{t}$ $B_{t+1}$.

3. $\kappa=1$ iff for all $t$ and disjoint $A_{t+1}, B_{t+1}, A_{t+1} \succsim_{t} B_{t+1}$ implies $A_{t+1} \succsim_{t} A_{t+1} \cup$ $B_{t+1} \succsim_{t} B_{t+1}$.

\footnotetext{
${ }^{13}$ We can also prove the following result: suppose that $P_{t+1}\left[a_{t+1} \mid\left\{a_{t+1}, h_{t+1}\right\}\right]=\varepsilon=$ $P_{t+1}\left[l_{t+1} \mid\left\{a_{t+1}, l_{t+1}\right\}\right]$. Then, for any $\varepsilon$, there exists $\kappa^{*}$ such that $\left\{a_{t+1}\right\} \succ_{t}\left\{h_{t+1}, l_{t+1}\right\}$ for $\kappa>\kappa^{*}$ and $\left\{a_{t+1}\right\} \prec_{t}\left\{h_{t+1}, l_{t+1}\right\}$ for $\kappa<\kappa^{*}$. Moreover, for any $\kappa$, there exists $\varepsilon^{*}$ such that $\left\{a_{t+1}\right\} \succ_{t}\left\{h_{t+1}, l_{t+1}\right\}$ for $\varepsilon>\varepsilon^{*}$ and $\left\{a_{t+1}\right\} \prec_{t}\left\{h_{t+1}, l_{t+1}\right\}$ for $\varepsilon<\varepsilon^{*}$.
} 
4. $\kappa \leq 0$ iff for all $t, A_{t+1} \succsim_{t} B_{t+1}$ whenever $A_{t+1} \supseteq B_{t+1}$.

5. $\kappa>0$ iff for all $t$ and for any $A_{t+1}$, there exists $b_{t+1} \notin A_{t+1}$ such that $A_{t+1} \succ_{t}$ $A_{t+1} \cup\left\{b_{t+1}\right\}$.

Of course, data on a finite set of menus could never prove that the representation also fit choices from menus that have not been observed. For this reason, our representation theorem in Section 6 places restrictions on choice from every menu; the theorem tells us just what conditions these choices must satisfy to be consistent with the representation.

\subsection{Immediate versus Delayed Consequences}

Dynamic choice problems also let us study how choices depend on whether their consequences are immediate or delayed. For example, suppose that the decision maker chooses $x$ with probability 0.51 from the menu $\{x, y\}$, as in Figure 4. What will the decision maker do if asked to make the choice between $x$ and $y$ one period before they can be consumed, that is, what is the probability of choosing the action $a=(z,\{x\})$ over $b=(z,\{y\})$ ? As noted above, DAL assigns shocks to all actions, even those without any immediate payoff consequences. At the time of the decision, the agent does not know the particular shocks that apply to $x$ and $y$ tomorrow. However, even though the menu $\{x\}$ is in expectation strictly better than $\{y\}$, the agent's choice between $a$ and $b$ is stochastic, as both actions receive independent payoff shocks today.

One obvious alternative model would be one where shocks are only to consumption. With the usual i.i.d. shocks assumption, that model predicts deterministic choice regardless of the real time that elapses between the choice and its consumption consequence in the next period. This stark conclusion strikes us as a significant drawback. The intermediate case where information about consumption shocks is gradually revealed over time would yield less stark predictions, but it seems too complicated to work with except in extremely simple problems.
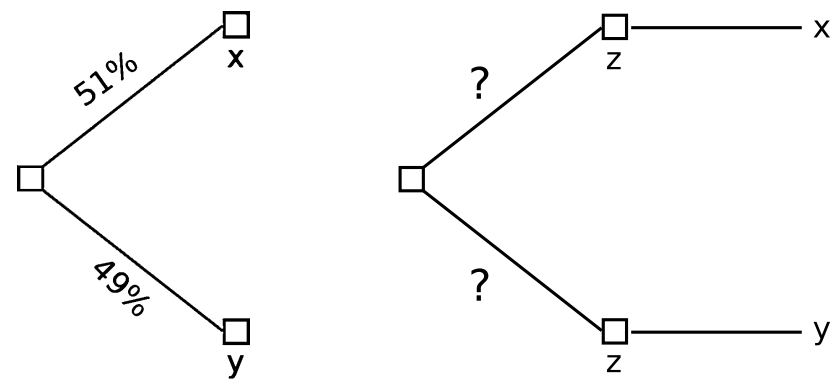

FIGURE 4.- Stochastic choice with delayed consumption. 


\subsection{Horizon Effects}

A related phenomenon is the relationship between the length of the delay and choice. How do the choice probabilities change when the consequences recede into the future? Are choices over later rewards more random than choices over sooner rewards? What happens in the limit?

Let $A$ be a subset of $Z$. Suppose the agent will receive a fixed sequence $\bar{z}=\left(z_{0}, z_{1}, \ldots, z_{T-1}\right)$ in periods 0 through $T-1$, and that the only nontrivial decision (nonsingleton choice problem) that the agent faces is to decide in time 0 which $\tilde{z}_{T}$ to receive at time $T$. Thus, the agent's time- 0 decision problem is to choose between $|A|$ different continuation problems, one for each element of $A$. Let $A_{0}:=\left\{\left(z_{0}, z_{1}, \ldots, z_{T-1}, \tilde{z}_{T}\right): \tilde{z}_{T} \in A\right\}$ be the choice set at time 0 . The object of interest is $P_{0}\left[\left(z_{0}, z_{1}, \ldots, z_{T-1}, \tilde{z}_{T}\right) \mid A_{0}\right]$, the choice probability of a given element $\tilde{z}_{T}$.

Discounting implies that the agent becomes less concerned about a choice as its consequences recede into the future, and so under DAR in the limit choice over distant rewards is close to the uniform distribution. To see that, note that if the agent picks $\tilde{z}_{T}$, his period- $T$ utility is $v\left(\tilde{z}_{T}\right)$, and his value in period 0 is $U_{0}\left(z_{0}, z_{1}, \ldots, z_{T-1}, \tilde{z}_{T}\right)=\sum_{t=0}^{T-1} \delta^{t} v\left(z_{t}\right)+\delta^{T} v\left(\tilde{z}_{T}\right)$. Thus, the agent's choice at time 0 is

$$
\begin{aligned}
P_{0}\left[\left(z_{0}, z_{1}, \ldots, z_{T-1}, \tilde{z}_{T}\right) \mid A_{0}\right] & =\frac{\exp \left(\sum_{t=0}^{T-1} \delta^{t} v\left(z_{t}\right)+\delta^{T} v\left(\tilde{z}_{T}\right)\right)}{\sum_{z^{\prime} \in A_{0}} \exp \left(\sum_{t=0}^{T-1} \delta^{t} v\left(z_{t}\right)+\delta^{T} v\left(z^{\prime}\right)\right)} \\
& =\frac{\exp \left(\delta^{T} v\left(\tilde{z}_{T}\right)\right)}{\sum_{z^{\prime} \in A_{0}} \exp \left(\delta^{T} v\left(z^{\prime}\right)\right)} .
\end{aligned}
$$

Note that even though the initial differences between the utilities of items of $A$ may be large, discounting makes them closer to each other, which leads to more uniform choice probabilities. In the limit, as $T \rightarrow \infty$ we have $P_{0}\left[\left(z_{0}, z_{1}, \ldots, z_{T-1}, \tilde{z}_{T}\right) \mid A_{0}\right] \rightarrow \frac{1}{|A|}$.

\subsection{Choosing When to Choose}

A concomitant question about timing is when the agent would like to make a choice from a given menu, with the outcome to be received at some later time.

Let $A$ be a subset of $Z$ with a generic element $\tilde{z}_{T}$. Suppose that the agent must choose between $a_{0}=\left(z_{0}, A_{1}\right)$ and $b_{0}=\left(z_{0}, B_{1}\right)$ at time 0 . Under either decision problem, he will receive the same sequence $\left(z_{1}, \ldots, z_{T-1}\right)$ in periods 0 through $T-1$. Under $A_{1}$, he will face a choice in period 1 of which element 


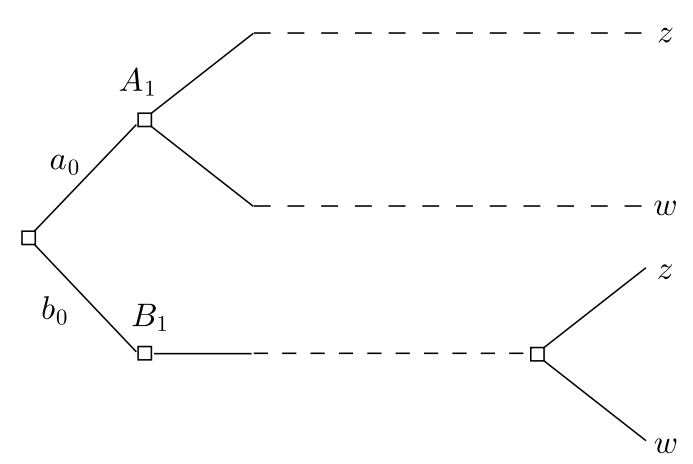

FIGURE 5.-Choosing when to choose with $A=\{z, w\}$.

$\tilde{z}_{T}$ to receive at time $T$, while under $B_{1}$, he selects his time- $T$ outcome $\tilde{z}_{T} \in A$ in period $T$. Figure 5 shows a simple problem of this kind; we are interested whether $a_{0} \succ_{0} b_{0}$ or vice versa.

The following result shows that the timing decisions of the agent depend on his choice aversion parameter $\kappa$ : agents who are more choice-loving have a stronger preference for making earlier decisions; agents who are more choiceaverse have a stronger preference for making later decisions.

\section{PROPOSITION 5:}

1. Fix any two DAR choice rules $(v, \delta, \kappa)$ and $\left(v, \delta, \kappa^{*}\right)$. If $a_{0} \succsim_{0} b_{0}$ and $\kappa>\kappa^{*}$, then $a_{0} \succsim_{0}^{*} b_{0}$. If $b_{0} \succsim_{0} a_{0}$ and $\kappa^{*}>\kappa$, then $b_{0} \succsim_{0}^{*} a_{0}$.

2. If $\kappa \leq 0$, then $a_{0} \succ_{0} b_{0}$ for any menu A. If $\kappa \geq 1$, then $b_{0} \succsim_{0} a_{0}$ for any menu (with indifference if and only if $\kappa=1$ and all the elements of the set $A$ are chosen with equal probabilities).

An agent with $\kappa=0$ always likes to make an early choice. Under DAE, she derives a benefit (measured by the entropy function) from the simple act of choice, and impatience implies she would like to receive this benefit as early as possible. Under DAL, the reason the agent prefers early resolution when $\kappa=0$ is that the payoff shocks $\varepsilon_{t}$ apply to pairs $\left(z_{t}, A_{t+1}\right)$ of current action and continuation plan, and since the expected value of the shock of the chosen action is positive, the agent again prefers early choice. This is a consequence of two key assumptions: first, that there are utility shocks to actions as opposed to shocks to consumption utility (as is needed to generate stochastic choice over any act that involves delayed consumption, such as purchasing cans of tuna fish), and second, that the desirability of future menus includes all of the "option value" that the utility shocks suggest. The adjustment embodied in the parameter $\kappa$ lets the model keep the analytic simplicity of i.i.d. shocks to actions while reducing or eliminating this option value. For example, when $\kappa \geq 1$, the menu-size adjustment is so large that the agent perceives the act of choice 
as a "bad." Then, because the agent is impatient and so prefers to postpone losses, a $\kappa \geq 1$ agent has a preference for later choice. This provides a way to identify $\kappa$ without using menus of different sizes (again on the assumption that the representation applies).

Some additional intuition for the link between $\kappa$ and preference over the timing of choice can be obtained by noticing that the value function in DALu expresses the value today as a nonlinear function of the choice probabilities tomorrow. This function resembles the formulation of Kreps and Porteus (1978) and especially the functional form of Epstein and Zin (1989), with the difference that in those models the randomization is exogenous, while here the randomization is a consequence of the actions of the agent herself. When $\kappa=1$, our agent has preferences toward late resolution of uncertainty because the nonlinear transformation of the probabilities is concave; here the adjusted logsum formula can be interpreted as a certainty equivalent.

The "shocks to actions" assumption, along with our other conditions, implies that agents who tend to like bigger menus are also more inclined to make decisions earlier, while agents who typically like smaller menus are also inclined to postpone their decisions. In contrast, the "shocks to immediate outcomes" model discussed in Section 3 implies that agents always at least weakly prefer larger menus and later choices. There are many possible intuitions for either case and they apply in different situations. Although many economists find preference for both larger menus and later choice intuitive, we personally would sometimes strictly prefer a menu with one great choice to a menu with the great choice and several awful ones, due to our awareness of our limited cognition: It takes time and effort to make choices, and sometimes we make mistakes. ${ }^{14}$ On the other hand, in some cases we might prefer larger menus for reasons outside of the model, such as getting utility from thinking about possible vacation plans. Likewise, there are cases where we prefer to make decisions early to get the decision over with (which fits with $\kappa<1$ ) or to avoid the need to remember to make it (which does not fit into our model), and cases where we prefer to postpone decisions to get more information or to avoid facing unpleasant alternatives. Moreover, there are other forces, such as regret aversion, that can lead to a preference for either larger or smaller menus and either sooner or later decisions. Unfortunately, we do not know of any empirical evidence on the prevalence of the preference for each of the four combinations (larger/smaller, earlier/later); such data would be a valuable guide to future work in this area.

\footnotetext{
${ }^{14}$ For example, the agent in the Rubinstein and Salant (2006) model of choice from lists can strictly prefer $\{x\}$ to $\{x, y\}$ because with the larger menu, if $y$ is presented first, he might end up choosing it despite $x$ being better.
} 


\section{AXIOMATIZATION}

We present the axioms in three subsections. The axioms in the first subsection simply ensure that preferences reduce to the logit case in a static problem and that preferences are independent of any fixed continuation problem, which also implies that preferences over today's outcomes with a fixed continuation problem reduce to logit. The second subsection develops axioms that relate choices at times $t$ and $t+1$, and pin down how the agent feels about replicating the objects in each menu. These axioms are sufficient to obtain recursive versions of DAR, in which choice at time $t$ depends on the utility of time- $t$ outcomes and a continuation value. However, just as in deterministic dynamic choice (Koopmans (1960)), additive, stationary, impatient representations require additional assumptions that we develop in the third subsection.

\subsection{Logit-esque Axioms}

AxIOM 1-Positivity: For any $t, A_{t} \in \mathcal{M}_{t}$, and $a_{t} \in A_{t}$, we have $P_{t}\left[a_{t} \mid A_{t}\right]>0$.

As argued by McFadden (1973), a zero probability is empirically indistinguishable from a positive but small probability, and since keeping all probabilities positive facilitates estimation, the positivity axiom is usually assumed in econometric analysis of both static and dynamic discrete choice. In settings where the stochastic term arises from random utility, positivity corresponds to the assumption that the utility shocks have sufficiently large support that even a typically unattractive option is occasionally preferred. Positivity is implied by the perturbed objective function representation for stochastic choice when the gradient of the perturbation becomes infinite on the boundary of the probability simplex; it has been motivated there by the fact that no deterministic rule can be Hannan (or "universally") consistent.

AXIOM 2-Stage IIA: For any $t \leq T, a_{t}, b_{t} \in \mathcal{A}_{t}$, and $A_{t}, B_{t} \in \mathcal{M}_{t}$ such that $a_{t}, b_{t} \in A_{t} \cap B_{t}$,

$$
\frac{P_{t}\left[a_{t} \mid A_{t}\right]}{P_{t}\left[b_{t} \mid A_{t}\right]}=\frac{P_{t}\left[a_{t} \mid B_{t}\right]}{P_{t}\left[b_{t} \mid B_{t}\right]},
$$

whenever the probabilities in the denominators are both positive.

Stage IIA says that the ratio of choice probabilities between two actions does not depend on other actions in the menu; it reduces to the standard IIA axiom in period $T$ by our assumption that choices do not depend on past history. Notice that positivity and IIA imply that the stochastic preference $\succsim_{t}$ is transitive (see, e.g., Luce (1959)). As is well known, this axiom is very restrictive. As we noted in the Introduction, it and the closely related logistic choice rule are widely used in empirical work for reasons of tractability. Assuming IIA lets 
us focus on other aspects of stochastic dynamic choice; we discuss some of the issues related to relaxing this assumption in Section 7.

AxIOM 3-Ordinal Time Separability: For all $t<T, x, y \in Z$, and $A_{t+1}$, $B_{t+1} \in \mathcal{M}_{t+1}$,

1. $\left(x, A_{t+1}\right) \succsim_{t}\left(x, B_{t+1}\right)$ iff $\left(y, A_{t+1}\right) \succsim_{t}\left(y, B_{t+1}\right)$.

2. $\left(x, A_{t+1}\right) \succsim_{t}\left(y, A_{t+1}\right)$ iff $\left(x, B_{t+1}\right) \succsim_{t}\left(y, B_{t+1}\right)$.

This axiom says that preferences over future decision problems are independent of the outcome in the current period, and conversely, that preferences over current outcomes do not depend on the choice problem to be confronted tomorrow. ${ }^{15}$ It is thus a stochastic version of Postulate 3 of Koopmans (1960), and corresponds to what Fishburn (1970, Chapter 4) called independence. Axiom 3 together with the recursivity axiom of Section 6.2 is sufficient for a history-independent recursive representation of the agent's preferences (see Lemma 5 in the Appendix).

\subsection{Tying Choices in Different Time Periods}

Now we introduce axioms that relate choices in period $t$ to choices and menus in the consecutive time period. The next axiom requires that future choice problem $A$ is more likely to be selected now than some other $B$ if elements of $A$ are more likely to be selected than elements of $B$ when both are presented as an immediate decision next period. Note well that this axiom only applies when $A$ and $B$ have the same size-for this reason, it does not constrain how the agent feels about adding items to a menu.

AXIOM 4-Recursivity: For all $t$ and menus $A_{t+1}, B_{t+1} \in \mathcal{M}_{t+1}$ with $\left|A_{t+1}\right|=$ $\left|B_{t+1}\right|$,

$$
A_{t+1} \succsim_{t} B_{t+1} \quad \text { iff } \quad P_{t+1}\left[A_{t+1} \mid A_{t+1} \cup B_{t+1}\right] \geq P_{t+1}\left[B_{t+1} \mid A_{t+1} \cup B_{t+1}\right] .
$$

We need additional axioms to pin down choices across menus of different sizes. Before stating these axioms, we present some others that help shed light on the role of $\kappa$ in the representations. The next axiom simply drops the qualifier $\left|A_{t+1}\right|=\left|B_{t+1}\right|$ from recursivity:

AxIOM-Aggregate Recursivity: For all $t$ and menus $A_{t+1}, B_{t+1} \in \mathcal{M}_{t+1}$,

$$
A_{t+1} \succsim_{t} B_{t+1} \quad \text { iff } \quad P_{t+1}\left[A_{t+1} \mid A_{t+1} \cup B_{t+1}\right] \geq P_{t+1}\left[B_{t+1} \mid A_{t+1} \cup B_{t+1}\right] .
$$

\footnotetext{
${ }^{15}$ Since at least one reader asked us whether Axioms 1 and 2 imply Axiom 3, we point out that Axiom 3 fails if preference has logistic choice with the following specification: $P\left[\left(z, A_{t+1}\right) \mid A_{t}\right]=U_{t}\left(z, A_{t+1}\right) / \sum_{\left(x, B_{t+1}\right) \in A_{t}} U_{t}\left(x, B_{t+1}\right)$ and $U_{t}\left(z, A_{t+1}\right)=v\left(z_{t}\right)+$ $\delta \sum_{\left(z_{t+1}, A_{t+2}\right) \in A_{t+1}} 2 \cdot \mathbb{1}_{\left\{z_{t+1}=z_{t}\right\}} u\left(z_{t+1}, A_{t+2}\right)$ for some functions $u$ and $v$.
} 
This axiom is satisfied by DAR with $\kappa=0$. At first sight, it might seem to require no more than that the agent is sophisticated, as it is a stochastic version of the temporal consistency axiom of Kreps and Porteus (1978), which requires that a future choice problem $A$ is selected now over some other $B$ if there exists an element of $A$ which is selected over any element of $B$ when both are presented as an immediate decision next period. ${ }^{16}$

Another noteworthy case is $\kappa=1$, which corresponds to the discounted relative entropy model. This is captured by "average recursivity," which says that choice problem $A$ is more likely to be selected now than some other $B$ if the average of the choice probabilities of elements of $A$ is higher than that of $B$ when the choice set tomorrow is the union of $A$ and $B$.

AXIOM-Average Recursivity: For all $t$ and menus $A_{t+1}, B_{t+1} \in M_{t+1}$,

$$
\begin{aligned}
& A_{t+1} \succsim_{t} B_{t+1} \quad \text { iff } \\
& \frac{1}{\left|A_{t+1}\right|} P_{t+1}\left[A_{t+1} \mid A_{t+1} \cup B_{t+1}\right] \geq \frac{1}{\left|B_{t+1}\right|} P_{t+1}\left[B_{t+1} \mid A_{t+1} \cup B_{t+1}\right] .
\end{aligned}
$$

We will now state an axiom that leads to a representation with an arbitrary value of $\kappa$. To do so, we need a technical condition to ensure that the domain of preference is suitably large.

AXIOM 5-Richness: For any $t \leq T$, action $\left(z, A_{t+1}\right) \in Z \times \mathcal{M}_{t+1}$, finite set of outcomes $Z^{\prime} \subseteq Z$, and $\lambda \in(0, \infty)$, there exists an outcome $z^{\lambda} \in Z \backslash Z^{\prime}$, such that

$$
\begin{aligned}
P_{t} & {\left[\left(z^{\lambda}, A_{t+1}\right) \mid\left\{\left(z, A_{t+1}\right),\left(z^{\lambda}, A_{t+1}\right)\right\}\right] } \\
& =\lambda P_{t}\left[\left(z, A_{t+1}\right) \mid\left\{\left(z, A_{t+1}\right),\left(z^{\lambda}, A_{t+1}\right)\right\}\right] .
\end{aligned}
$$

In the case $\lambda=1$ we say that $z$ and $z^{1}$ are "equivalents." By Richness, there are arbitrarily many equivalents of each outcome, and also many " $\lambda$-equivalents." An equivalent of an action $\left(z, A_{t+1}\right)$ is any action of the form $\left(z^{1}, A_{t+1}\right)$ where $z^{1}$ is an equivalent of $z$. Let $A_{t+1} \in \mathcal{M}_{t+1}$ and $n$ be an integer. We say that a menu $A_{t+1}^{\prime}$ is an $n$-replica of $A_{t+1}$ whenever, for each $a \in A_{t+1}$, the menu $A_{t+1}^{\prime}$ contains $n$ equivalents of $a$ and $\left|A_{t+1}^{\prime}\right|=n\left|A_{t+1}\right|$. We denote by $n * A_{t+1}$ any $n$-replica of $A_{t+1}$; each such replica is treated equivalently by the agent.

\footnotetext{
${ }^{16}$ The axiom is also similar to Koopmans' Postulate 4, which combines the requirement of stationarity with dynamic consistency.
} 
AXIOM 6-Replica Invariance: For any $t \leq T$, outcomes $x, y \in Z$, continuation menus $A_{t+1}, B_{t+1}$, and integer $n$,

$$
\frac{P_{t}\left[\left(x, A_{t+1}\right) \mid C_{t}\right]}{P_{t}\left[\left(x, B_{t+1}\right) \mid C_{t}\right]}=\frac{P_{t}\left[\left(y, n * A_{t+1}\right) \mid C_{t}\right]}{P_{t}\left[\left(y, n * B_{t+1}\right) \mid C_{t}\right]},
$$

where $C_{t}=\left\{\left(x, A_{t+1}\right),\left(x, B_{t+1}\right),\left(y, n * A_{t+1}\right),\left(y, n * B_{t+1}\right)\right\}$.

To understand this axiom, suppose that $A_{t+1}=\{a\}$ and $B_{t+1}=\{b, c\}$. Then, in period $t+1$, when choosing from $n * A_{t+1}$, the agent is sure to end up with an equivalent of $a$. On the other hand, the probability of choosing an equivalent of $b$ when choosing from $n * B_{t+1}$ is the same as the probability of choosing $b$ from $B_{t+1}$ (as a consequence of Stage IIA), so that the probability ratio of $b$ and $c$ is the same whether they are being chosen from $B_{t+1}$ or from $n * B_{t+1}$. Thus the only reason that $\frac{P_{t}\left[\left(x, A_{t+1}\right) \mid C_{t}\right]}{P_{t}\left[\left(x, B_{t+1}\right) \mid C_{t}\right]}$ might differ from $\frac{P_{t}\left[\left(y, n * A_{t+1}\right) \mid C_{t}\right]}{P_{t}\left[\left(y, n * B_{t+1}\right) \mid C_{t}\right]}$ is because of the effect of menu size per se: For example, if the agent likes menus of size 1 much more than those of any other size, it might be that $\frac{P_{t}\left[\left(x, A_{t+1}\right) \mid C_{t}\right]}{P_{t}\left[\left(x, B_{t+1}\right) \mid C_{t}\right]}>\frac{P_{t}\left[\left(y, n * A_{t+1}\right) \mid C_{t}\right]}{P_{t}\left[\left(y, n * B_{t+1}\right) \mid C_{t}\right]}$. The axiom rules this out, and requires instead that the menu-size effect cancels out.

\subsection{Stationarity and Impatience}

To obtain a more specific and more tractable representation, we first impose an axiom that ensures that the effect of moving a decision from period $t+1$ to period $t$ depends only on the period- $t+1$ probabilities, and not the identities of the actions.

AXIOM 7-Probability Equivalence: For any $t<T, a_{t+1}, b_{t+1}, c_{t+1}, d_{t+1} \in$ $\mathcal{A}_{t+1}$, and $x, y \in Z$,

$$
P_{t+1}\left[a_{t+1} \mid\left\{a_{t+1}, b_{t+1}\right\}\right]=P_{t+1}\left[c_{t+1} \mid\left\{c_{t+1}, d_{t+1}\right\}\right]
$$

iff

$$
\begin{aligned}
P_{t} & {\left[\left(x,\left\{a_{t+1}\right\}\right) \mid\left\{\left(x,\left\{a_{t+1}\right\}\right),\left(x,\left\{b_{t+1}\right\}\right)\right\}\right] } \\
& =P_{t}\left[\left(y,\left\{c_{t+1}\right\}\right) \mid\left\{\left(y,\left\{c_{t+1}\right\}\right),\left(y,\left\{d_{t+1}\right\}\right)\right\}\right] .
\end{aligned}
$$

The axioms we have developed so far are sufficient to characterize a version of DAR where the value is a discounted sum but where various terms such as the discount factor or utility functions can vary over time. To obtain a stationary discounted model, we also need an axiom to ensure that the discount factor and utility functions are time invariant. The original form of stationarity introduced by Koopmans (1960) relies on an infinite horizon; we use a similar 
axiom of Fishburn (1970, Chapter 7). The axiom is imposed on the period-zero preference over consumption streams $\left(z_{0}, z_{1}, \ldots, z_{T}\right)$ that is induced from $\succsim_{0}$ by appropriately defining $A_{T}:=\left\{z_{T}\right\}$, and recursively $A_{t-1}=\left\{\left(z_{t-1}, A_{t}\right)\right\}$ for $t=T-1, \ldots, 0$.

AXIOM 8-Stream Stationarity: For any $z, z_{1}, \ldots, z_{T}, z_{1}^{\prime}, \ldots, z_{T}^{\prime} \in Z$,

$$
\begin{aligned}
& \left(z, z_{1}, \ldots, z_{T}\right) \succsim_{0}\left(z, z_{1}^{\prime}, \ldots, z_{T}^{\prime}\right) \quad \text { iff } \\
& \left(z_{1}, \ldots, z_{T}, z\right) \succsim_{0}\left(z_{1}^{\prime}, \ldots, z_{T}^{\prime}, z\right) .
\end{aligned}
$$

In conjunction with the previous axioms, the next axiom ensures that the way the agent chooses between menus of different cardinality does not depend on the time period.

AXIOM 9-Stationary Choice Aversion: For any $t<T$, outcome $z \in Z$, and continuation menus $A_{1} \in \mathcal{M}_{1}$ and $A_{t+1} \in \mathcal{M}_{t+1}$,

$$
\begin{aligned}
& P_{0}\left[\left(z, A_{1}\right) \mid\left\{\left(z, A_{1}\right),\left(z, 2 * A_{1}\right)\right\}\right] \\
& \quad=P_{t}\left[\left(z, A_{t+1}\right) \mid\left\{\left(z, A_{t+1}\right),\left(z, 2 * A_{t+1}\right)\right\}\right] .
\end{aligned}
$$

Finally, Impatience says that the agent prefers receiving better outcomes earlier on and so ensures that the discount factor is less than 1.

AXIOM 10-Impatience: For any $z, z^{\prime}, z_{0}, \ldots, z_{T} \in Z$, if $(z, \ldots, z) \succ_{0}$ $\left(z^{\prime}, \ldots, z^{\prime}\right)$, then

$$
\left(z_{0}, \ldots, z_{t-1}, z, z^{\prime}, z_{t+2}, \ldots, z_{T}\right) \succsim_{0}\left(z_{0}, \ldots, z_{t-1}, z^{\prime}, z, z_{t+2}, \ldots, z_{T}\right) .
$$

\subsection{Representation Theorem}

THEOREM 1: Suppose that P satisfies Richness. Then P satisfies Positivity, Stage IIA, Ordinal Time Separability, Recursivity, Replica Invariance, Probability Equivalence, Stream Stationarity, Stationary Choice Aversion, and Impatience if and only if it has a DAR.

As the following corollary shows, by strengthening Recursivity to Aggregate Recursivity (and dropping Stationary Choice Aversion and Replica Invariance), we obtain an axiomatization of the standard Discounted Logit functional form.

Corollary 1: Suppose that P satisfies Richness. Then P satisfies Positivity, Stage IIA, Ordinal Time Separability, Aggregate Recursivity, Probability Equivalence, Stream Stationarity, and Impatience if and only if it has a Discounted Logit representation. 
COROLlary 2: Suppose that P satisfies Richness. Then P satisfies Positivity, Stage IIA, Ordinal Time Separability, Average Recursivity, Probability Equivalence, Stream Stationarity, and Impatience if and only if it has a Discounted Entropy representation with $\kappa=1$.

The proof of Theorem 1 follows three main steps.

STEP 1: Lemma 4 shows that Axioms 1-3 are equivalent to a "sequential Luce representation": there are weights $W_{t}$ for actions $a_{t}$ such that

$$
P_{t}\left[a_{t} \mid A_{t}\right]=\frac{W_{t}\left(a_{t}\right)}{\sum_{b_{t} \in A_{t}} W_{t}\left(b_{t}\right)} .
$$

Here our maintained assumption that $P_{t}$ is history independent and Axioms 1 and 2 let us use Luce's original argument to conclude there are weights that describe period- $t$ choice, and Axiom 3 then lets us mimic the proof of Koopmans' Proposition 3 and conclude that $W_{t}\left(z_{t}, A_{t+1}\right)=G_{t}\left(v_{t}(z), h_{t}\left(A_{t+1}\right)\right)$, where $G_{t}$ is a strictly increasing function of the utility $v_{t}\left(z_{t}\right)$ and "anticipated utility" $h_{t}\left(A_{t+1}\right)$.

STEP 2: Lemma 5 shows that adding Recursivity implies that the function $h_{t}$ is of the form

$$
h_{t}\left(A_{t+1}\right)=f_{t}^{\left|A_{t+1}\right|}\left(\sum_{a_{t+1} \in A_{t+1}} W_{t+1}\left(a_{t+1}\right)\right),
$$

where $f_{t}^{n}$ is a family of increasing functions that depend on the cardinality $n$ of the menu.

STEP 3: The final step is to show that Richness, Replica Invariance, and Probability Equivalence imply that $P$ has a DAR with time-dependent parameters $\left(v_{t}, \delta_{t}, \kappa_{t}\right)$. Finally, Stream Stationarity, Stationary Choice Aversion, and Impatience imply that the parameters are time invariant.

\section{DISCUSSION}

\subsection{Relation to the Literature}

The paper is related to quite a large number of others, as it draws on and extends the literature on static stochastic choice pioneered by Luce (1959), Marschak (1959), and Harsanyi (1973a), the literature on discounting representations of deterministic dynamic choice (notably Koopmans (1960)), and the literature on choices over menus pioneered by Kreps (1979). 
We have maintained the widely used but widely criticized IIA assumption. While empirical analyses of dynamic choice continue to use IIA, empirical work on static choice uses alternatives such as nested logit and BLP (Berry, Levinsohn, and Pakes (1995)) that avoid some of IIA's starkest implications. Similarly, the theoretical work of Falmagne (1978), Barberá and Pattanaik (1986), Gul and Pesendorfer (2006), and Gul, Natenzon, and Pesendorfer (2014) provides axiomatic characterizations of static random utility models without the IIA assumption. Gul, Natenzon, and Pesendorfer (2014) is of particular note here, as they characterized an analog of nested logit that allows for items to be perfect, as opposed to almost-perfect, substitutes, which eliminates any "option value" that arises from adding exact substitutes to a menu. Moreover, in a recent addition to their paper, they extended their characterization to dynamic choice problems in which consumption occurs in a single time period and the agent is completely patient and so insensitive to the timing of payoffs and decisions. Fudenberg, Iijima, and Strzalecki (2014) provided axiomatic characterizations of stochastic choice by an ambiguity-averse agent as a form of nonlinear perturbed utility without assuming IIA, thus generalizing the entropy functional form to other perturbations; they also provided a characterization of nested logit. This work suggests that our dynamic representations could also be generalized beyond IIA, though obtaining a model of recursive choice problems that is both general and tractable seems challenging.

In recent years, there have been several generalizations of Koopmans' (1960) characterization to forms of "behavioral" dynamic choice, as in Jackson and Yariv (2010) and Montiel Olea and Strzalecki (2014); introducing stochastic choice into those setups could be a useful tool for analyzing experimental results.

The most active related literature is that on choice between menus. Some of these papers develop representations motivated by "consideration costs" or "costs of thinking"; to the extent that this cost is increasing in the menu size, it is related to our representations. Ergin and Sarver (2010), following Ergin (2003), developed a representation with a double maximization, in which "costly contemplation" corresponds to buying a signal about the second-period attractiveness of the various options. Though their primitives did not include lotteries over menus, they motivated their work with the idea that the agent prefers that such lotteries are resolved before she chooses an alternative so that she can avoid formulating a complete contingent plan. This motivation seems related to our comparative statics (Proposition 5) about choice aversion and preference for late decisions. Ortoleva (2013) explicitly considered lotteries and developed a model of "cost of thinking" where the agent ranks lotteries over menus as if she expected to choose the best option from each of them.

Other recent papers on choice from menus are of interest here primarily for how they impose recursivity or dynamic consistency. Ahn and Sarver (2013) is perhaps closest, as, like this paper, it treats both initial choice of a menu and subsequent choice from it as observable. They used recursivity axioms to 
pin down a unique state space and probabilities in the two-stage menu choice model; their Axiom 1 is similar in spirit to our Aggregate Recursivity condition, but as stated, it is vacuously satisfied given our positivity assumption. ${ }^{17}$ Krishna and Sadowski (2014) provided two representations for a decision maker who is uncertain about his future utility in an infinite-horizon decision problem. Their stationarity axiom corresponds to our Axioms 3 and 8, but Recursivity is inconsistent with the indifference required by their "continuation strategic rationality" axiom, though they did assume the agent prefers larger menus.

The paper is also related to the use of nonlinear perturbed utility in the theory of nonequilibrium learning in static games, as in Fudenberg and Levine (1995), Hart and Mas-Colell (2001), Hofbauer and Hopkins (2005), Hofbauer and Sandholm (2002), and Fudenberg and Takahashi (2011). Fudenberg and Levine (1995) showed that this generates a choice rule that is Hannan consistent, meaning that the decision maker gets at least the payoff that would be obtained from maximizing against the long-run average of play by Nature and any other players (Hannan (1957)). One motivation for this paper is to extend that work to allow for dynamic considerations, such as would arise in learning to play an extensive-form game.

\subsection{Conclusion}

This paper provides an axiomatic characterization of three equivalent generalizations of discounted logit, namely discounted adjusted logit, discounted adjusted entropy, and discounted adjusted Luce. These representations include a choice aversion parameter that captures the link between choices in different periods by adjusting the implied "option value" of larger menus. We point out that discounted logit is the special case of $\kappa=0$, where the agent always prefers adding any item to any menu. In this case, the agent also has a preference for early decision, which highlights the fact that a preference for larger menus can arise for many reasons, of which Kreps' (1979) preference for flexibility as usually understood is only one; this indicates the benefit of considering a richer decision domain than that in Kreps (1979). The more general discounted adjusted logit penalizes larger menus and so reduces the value of making early decisions: As $\kappa$ increases, the agent is less attracted to larger menus and more inclined to delay decisions, and when $\kappa \geq 1$, the agent always prefers to put decisions off.

\footnotetext{
${ }^{17} \mathrm{Ahn}$ and Sarver assumed a preference for larger choice sets and so ruled out temptation. Dekel and Lipman (2012) imposed consistency between the first-period choice of a menu and second-period choice from a menu at the level of the representation, and used choices in the two periods to distinguish between "random GP" and "random Strotz" representations in cases where temptation is present. They also showed that the random Strotz model can accommodate the nonlinear cost of self control introduced by Fudenberg and Levine (2006) and further analyzed by Fudenberg and Levine $(2011,2012)$ and Noor and Takeoka (2010a, 2010b).
} 
The axiomatic characterization of our model provides a foundation for the inclusion of a choice aversion parameter in empirical work. This representation is just as tractable as the usual discounted logit and may better describe behavior in at least some choice problems where the menu size varies.

\section{APPENDIX}

\section{A.1. Proof of Proposition 1}

To prove the proposition, we use the well-known following lemmas. The first lemma can be found in Anderson, De Palma, and Thisse (1992, Section 3.6).

LEMMA 1: For any vector $\left(x_{1}, \ldots, x_{n}\right) \in \mathbb{R}^{n}$,

$$
\begin{gathered}
\max _{p \in \Delta^{n}} \sum_{i=1}^{n} p_{i} x_{i}+H(p)=\log \left(\sum_{i=1}^{n} \exp \left(x_{i}\right)\right) \\
\text { and the solution is } \quad p_{i}=\frac{\exp \left(x_{i}\right)}{\sum_{j=1}^{n} \exp \left(x_{j}\right)} .
\end{gathered}
$$

The following lemma is an easy conclusion from Lemma 1.

LEMMA 2: For any vector $\left(x_{1}, \ldots, x_{n}\right) \in \mathbb{R}^{n}$,

$$
\begin{gathered}
\max _{p \in \Delta^{n}} \sum_{i=1}^{n} p_{i} x_{i}+J_{\kappa}^{n}(p)=\log \left(\sum_{i=1}^{n} \exp \left(x_{i}\right)\right)-\kappa \log n \\
\text { and the solution is } p_{i}=\frac{\exp \left(x_{i}\right)}{\sum_{j=1}^{n} \exp \left(x_{j}\right)} .
\end{gathered}
$$

The next lemma can be found in Train (2009, Chapter 3).

LEMMA 3: Suppose that $\varepsilon_{1}, \ldots, \varepsilon_{n}$ are i.i.d. random variables with the extreme value distribution with noise level parameter 1 . For any vector $\left(x_{1}, \ldots, x_{n}\right) \in \mathbb{R}^{n}$,

$$
\begin{aligned}
& \mathbb{E}\left[\max _{i=1, \ldots, n} x_{i}+\varepsilon_{i}\right]=\log \left(\sum_{i=1}^{n} \exp \left(x_{i}\right)\right) \text { and } \\
& \operatorname{Prob}\left[x_{i}+\varepsilon_{i} \geq \max _{j=1, \ldots, n} x_{j}+\varepsilon_{j}\right]=\frac{\exp \left(x_{i}\right)}{\sum_{j=1}^{n} \exp \left(x_{j}\right)} .
\end{aligned}
$$




\section{$D A L$ Equivalent to $D A L u$}

By Lemma 3, it follows that $\left\{P_{t}\right\}$ has a DAL representation iff

$$
U_{t}\left(z_{t}, A_{t+1}\right)=v\left(z_{t}\right)+\delta \log \left(\sum_{a_{t+1} \in A_{t+1}} \exp \left(U_{t+1}\left(a_{t+1}\right)\right)\right)-\delta \kappa \log \left|A_{t+1}\right|
$$

and

$$
P_{t}\left[a_{t} \mid A_{t}\right]=\frac{\exp \left(U_{t}\left(a_{t}\right)\right)}{\sum_{b_{t} \in A_{t}} \exp \left(U_{t}\left(b_{t}\right)\right)} .
$$

By letting $W_{t}=\exp \left(U_{t}\right)$, this is equivalent to a DALu representation.

\section{DAE Equivalent to DALu}

By Lemma 2, it follows that $\left\{P_{t}\right\}$ has a DAE representation iff

$$
U_{t}\left(z_{t}, A_{t+1}\right)=v\left(z_{t}\right)+\delta \log \left(\sum_{a_{t+1} \in A_{t+1}} \exp \left(U_{t+1}\left(a_{t+1}\right)\right)\right)-\delta \kappa \log \left|A_{t+1}\right|
$$

and

$$
P_{t}\left[a_{t} \mid A_{t}\right]=\frac{\exp \left(U_{t}\left(a_{t}\right)\right)}{\sum_{b_{t} \in A_{t}} \exp \left(U_{t}\left(b_{t}\right)\right)} .
$$

By letting $W_{t}=\exp \left(U_{t}\right)$, this is equivalent to a DALu representation.

\section{Uniqueness}

Suppose that $(v, \delta, \kappa)$ and $\left(v^{\prime}, \delta^{\prime}, \kappa^{\prime}\right)$ are both DALu representations of $\left\{P_{t}\right\}$. The induced period-zero choice between consumption streams $\tilde{z}=\left(z_{0}, \ldots, z_{T}\right)$ (for the definition of consumption streams, see axiom Stream Stationarity) has static Luce representations (Luce (1959)) $\tilde{z} \mapsto \exp \left(\sum_{t=0}^{T} \delta^{t} v\left(z_{t}\right)\right)$ and by $\tilde{z} \mapsto$ $\exp \left(\sum_{t=0}^{T} \delta^{t} v^{\prime}\left(z_{t}\right)\right)$. By uniqueness of Luce representations, there exists $\alpha>0$ such that $\exp \left(\sum_{t=0}^{T} \delta^{t} v\left(z_{t}\right)\right)=\alpha \exp \left(\sum_{t=0}^{T} \delta^{\prime t} v^{\prime}\left(z_{t}\right)\right)$ for all $\left(z_{0}, \ldots, z_{T}\right) \in Z^{T+1}$. By fixing $\left(\bar{z}_{1}, \ldots, \bar{z}_{T}\right) \in Z^{T}$, this implies that there exists a constant $\beta$ such that $v\left(z_{0}\right)=v^{\prime}\left(z_{0}\right)+\beta$ for all $z_{0} \in Z$. By fixing $\left(\bar{z}_{0}, \bar{z}_{2}, \ldots, \bar{z}_{T}\right) \in Z^{T}$, this implies that $\delta^{\prime}=\delta$. 
Finally, consider $A_{T-1}=\left\{(z,\{x\}),\left(z,\left\{y_{1}, y_{2}\right\}\right)\right\}$ for some arbitrary $x, y_{1}$, $y_{2}, z \in Z$. We have

$$
\begin{aligned}
\frac{P_{T-1}\left[(z,\{x\}) \mid A_{T-1}\right]}{P_{T-1}\left[\left(z,\left\{y_{1}, y_{2}\right\}\right) \mid A_{T-1}\right]} & =\frac{\exp (\delta v(x))}{\exp \left(\delta \log \left(e^{v\left(y_{1}\right)}+e^{v\left(y_{2}\right)}\right)-\delta \kappa \log 2\right)} \\
& =\frac{\exp (\delta v(x)+\delta \beta)}{\exp \left(\delta \log \left(e^{v\left(y_{1}\right)+\beta}+e^{v\left(y_{2}\right)+\beta}\right)-\delta \kappa^{\prime} \log 2\right)} \\
& =\frac{\exp (\delta v(x)+\delta \beta)}{\exp \left(\delta \log \left(e^{v\left(y_{1}\right)}+e^{v\left(y_{2}\right)}\right)+\delta \beta-\delta \kappa^{\prime} \log 2\right)} \\
& =\frac{\exp (\delta v(x))}{\exp \left(\delta \log \left(e^{v\left(y_{1}\right)}+e^{v\left(y_{2}\right)}\right)-\delta \kappa^{\prime} \log 2\right)}
\end{aligned}
$$

so $\kappa^{\prime}=\kappa$.

\section{A.2. Recursive Representations}

In this section, we study recursive representations à la Koopmans (1960) and Kreps and Porteus (1978). The representations we present here have fewer time-separability properties than a discounted sum and do not insist on stationarity, and therefore correspond to a shorter list of axioms. We use these representations as intermediate steps towards the main theorem.

DEFINITION 10-Sequential Luce: $\left\{P_{t}\right\}$ has a Sequential Luce Representation if there exist functions $v_{t}: Z \rightarrow \mathbb{R}, h_{t}: \mathcal{M}_{t+1} \rightarrow \mathbb{R}$ with ranges $R v_{t}$ and $R h_{t}$, respectively, and $G_{t}: R v_{t} \times R h_{t} \rightarrow \mathbb{R}_{++}$, strictly increasing in both variables, and value functions $W_{t}: \mathcal{A}_{t} \rightarrow \mathbb{R}_{++}$recursively defined by

$$
W_{t}\left(z_{t}, A_{t+1}\right)=G_{t}\left(v_{t}\left(z_{t}\right), h_{t}\left(A_{t+1}\right)\right),
$$

such that, for all $A_{t}$ and all $a_{t} \in A_{t}$,

$$
P_{t}\left[a_{t} \mid A_{t}\right]=\frac{W_{t}\left(a_{t}\right)}{\sum_{b_{t} \in A_{t}} W_{t}\left(b_{t}\right)} .
$$

Definition 11-Recursive Adjusted Luce: $\left\{P_{t}\right\}$ has a Recursive Adjusted Luce representation if it has a sequential Luce representation with

$$
h_{t}\left(A_{t+1}\right)=f_{t}^{\left|A_{t+1}\right|}\left(\sum_{a_{t+1} \in A_{t+1}} W_{t+1}\left(a_{t+1}\right)\right)
$$

for some family of increasing functions $f_{t}^{n}$. 
Lemma 4: P satisfies Positivity, Stage IIA, and Ordinal Time Separability iff it has a sequential Luce representation. If, in addition, it satisfies Richness, then $\left\{W_{t}\left(z_{t}, A_{t+1}\right) \mid z_{t} \in Z\right\}=(0, \infty)$ for any $A_{t+1} \in \mathcal{M}_{t+1}$.

Lemma 5: P satisfies Positivity, Stage IIA, Ordinal Time Separability, and Recursivity iff it has a recursive adjusted Luce representation.

\section{A.3. Proof of Lemma 4}

\section{A.3.1. Sufficiency}

STEP 1: Fix $\bar{z} \in Z$ and define $W_{T}(\bar{z}):=1$. For any other $z \in Z$, define

$$
W_{T}(z):=\frac{P_{T}[z \mid\{z, \bar{z}\}]}{P_{T}[\bar{z} \mid\{z, \bar{z}\}]} .
$$

For any $A_{T}=\left\{z^{1}, \ldots, z^{n}\right\} \in \mathcal{M}_{T}$, Positivity and Stage IIA imply that, for all $i, j=1, \ldots, n$,

$$
\frac{P_{T}\left[z^{i} \mid A_{T}\right]}{P_{T}\left[z^{j} \mid A_{T}\right]}=\frac{P_{T}\left[z^{i} \mid\left\{z^{i}, z^{j}, \bar{z}\right\}\right]}{P_{T}\left[z^{j} \mid\left\{z^{i}, z^{j}, \bar{z}\right\}\right]}=\frac{W_{T}\left(z^{i}\right)}{W_{T}\left(z^{j}\right)},
$$

so equation (8) holds. For $t \leq T$, we proceed analogously to define $W_{t}\left(z, A_{t+1}\right)$ that satisfies equation (8).

STEP 2: Part (1) of Ordinal Time Separability and Step 1 imply that, for all $z, z^{\prime} \in Z$ and $A_{t+1}, A_{t+1}^{\prime} \in \mathcal{M}_{t+1}$,

$$
W_{t}\left(z, A_{t+1}\right) \geq W_{t}\left(z, A_{t+1}^{\prime}\right) \quad \Longleftrightarrow W_{t}\left(z^{\prime}, A_{t+1}\right) \geq W_{t}\left(z^{\prime}, A_{t+1}^{\prime}\right) .
$$

Fix $\bar{z} \in Z$ and define $h_{t}\left(A_{t+1}\right):=W_{t}\left(\bar{z}, A_{t+1}\right)$ for all $A_{t+1} \in \mathcal{M}_{t+1}$. Property (10) implies that for any $z \in Z$, there is a strictly increasing function $f_{t}^{z}: R h_{t} \rightarrow \mathbb{R}$ such that $W_{t}\left(z, A_{t+1}\right)=f_{t}^{z}\left(h_{t}\left(A_{t+1}\right)\right)$ for all $A_{t+1} \in \mathcal{M}_{t+1}$. Define a real valued function $F_{t}: Z \times R h_{t} \rightarrow \mathbb{R}$ by $F_{t}(z, h)=f_{t}^{z}(h)$ for any $z \in Z$ and $h \in R h_{t}$. Note that the function $F_{t}$ is strictly increasing in the second variable. With this notation, we have $W_{t}\left(z, A_{t+1}\right)=F_{t}\left(z, h_{t}\left(A_{t+1}\right)\right)$.

STEP 3: Part (2) of Ordinal Time Separability and Steps 1-2 imply that, for all $z, z^{\prime} \in Z$ and $A_{t+1}, A_{t+1}^{\prime} \in \mathcal{M}_{t+1}$,

$$
\begin{aligned}
& F_{t}\left(z, h_{t}\left(A_{t+1}\right)\right) \geq F_{t}\left(z^{\prime}, h_{t}\left(A_{t+1}\right)\right) \\
& \quad \Longleftrightarrow \quad F_{t}\left(z, h_{t}\left(A_{t+1}^{\prime}\right)\right) \geq F_{t}\left(z^{\prime}, h_{t}\left(A_{t+1}^{\prime}\right)\right) .
\end{aligned}
$$

Fix $\bar{h} \in R h_{t}$ and define $v_{t}(z):=F_{t}(z, \bar{h})$. Property (11) implies that for any $h \in R h_{t}$, there exists a strictly increasing function $m_{t}^{h}: R v_{t} \rightarrow \mathbb{R}$ such that 
$F_{t}(z, h)=m_{t}^{h}\left(v_{t}(z)\right)$. Define a real valued function $G_{t}: R v_{t} \times R h_{t} \rightarrow \mathbb{R}$ by $G_{t}(v, h)=m_{t}^{h}(v)$ for any $v \in R v_{t}$ and $h \in R h_{t}$. Note that the function $G_{t}$ is strictly increasing in both variables. This implies equation (7). ${ }^{18}$

\section{A.3.2. Necessity}

Positivity is immediate since the function $W$ takes strictly positive values, and Stage IIA follows immediately from formula (8). To prove that the first part of Ordinal Time Separability holds, we need to show that

$$
\begin{aligned}
& P_{t}\left[\left(z, A_{t+1}\right) \mid\left\{\left(z, A_{t+1}\right),\left(z, A_{t+1}^{\prime}\right)\right\}\right] \\
& \quad \geq P_{t}\left[\left(z, A_{t+1}^{\prime}\right) \mid\left\{\left(z, A_{t+1}\right),\left(z, A_{t+1}^{\prime}\right)\right\}\right]
\end{aligned}
$$

iff

$$
\begin{aligned}
& P_{t}\left[\left(z^{\prime}, A_{t+1}\right) \mid\left\{\left(z^{\prime}, A_{t+1}\right),\left(z^{\prime}, A_{t+1}^{\prime}\right)\right\}\right] \\
& \quad \geq P_{t}\left[\left(z^{\prime}, A_{t+1}^{\prime}\right) \mid\left\{\left(z^{\prime}, A_{t+1}\right),\left(z^{\prime}, A_{t+1}^{\prime}\right)\right\}\right] .
\end{aligned}
$$

By formula (8), this is equivalent to $W_{t}\left(z, A_{t+1}\right) \geq W_{t}\left(z, A_{t+1}^{\prime}\right)$ iff $W_{t}\left(z^{\prime}, A_{t+1}\right) \geq$ $W_{t}\left(z^{\prime}, A_{t+1}^{\prime}\right)$. Since by formula (7), $W_{t}\left(z_{t}, A_{t+1}\right)=G_{t}\left(v_{t}\left(z_{t}\right), h_{t}\left(A_{t+1}\right)\right)$, where $G_{t}$ is increasing in its second argument, we know that $W_{t}\left(z, A_{t+1}\right) \geq W_{t}\left(z, A_{t+1}^{\prime}\right)$ iff $h_{t}\left(A_{t+1}\right) \geq h_{t}\left(A_{t+1}^{\prime}\right)$ iff $W_{t}\left(z^{\prime}, A_{t+1}\right) \geq W_{t}\left(z^{\prime}, A_{t+1}^{\prime}\right)$. To prove that the second part of Ordinal Time Separability holds, we need to show that

$$
\begin{aligned}
& P_{t}\left[\left(z, A_{t+1}\right) \mid\left\{\left(z, A_{t+1}\right),\left(z^{\prime}, A_{t+1}\right)\right\}\right] \\
& \quad \geq P_{t}\left[\left(z^{\prime}, A_{t+1}\right) \mid\left\{\left(z, A_{t+1}\right),\left(z^{\prime}, A_{t+1}\right)\right\}\right]
\end{aligned}
$$

iff

$$
\begin{aligned}
& P_{t}\left[\left(z, A_{t+1}^{\prime}\right) \mid\left\{\left(z, A_{t+1}^{\prime}\right),\left(z^{\prime}, A_{t+1}^{\prime}\right)\right\}\right] \\
& \quad \geq P_{t}\left[\left(z^{\prime}, A_{t+1}^{\prime}\right) \mid\left\{\left(z, A_{t+1}^{\prime}\right),\left(z^{\prime}, A_{t+1}^{\prime}\right)\right\}\right] .
\end{aligned}
$$

By formula (8), this is equivalent to $W_{t}\left(z, A_{t+1}\right) \geq W_{t}\left(z^{\prime}, A_{t+1}\right)$ iff $W_{t}\left(z, A_{t+1}^{\prime}\right) \geq$ $W_{t}\left(z^{\prime}, A_{t+1}^{\prime}\right)$. Since, by formula (7), $W_{t}\left(z_{t}, A_{t+1}\right)=G_{t}\left(v_{t}\left(z_{t}\right), h_{t}\left(A_{t+1}\right)\right)$, where $G_{t}$ is increasing in its first argument, we know that $W_{t}\left(z, A_{t+1}\right) \geq W_{t}\left(z^{\prime}, A_{t+1}\right)$ iff $v_{t}(z) \geq v_{t}\left(z^{\prime}\right)$ iff $W_{t}\left(z, A_{t+1}^{\prime}\right) \geq W_{t}\left(z^{\prime}, A_{t+1}^{\prime}\right)$.

\footnotetext{
${ }^{18}$ Note that our Steps 2 and 3 essentially mimic Koopmans' (1960) proof, which led him to formula (7).
} 


\section{A.3.3. Surjectivity}

Fix an arbitrary element $\left(z_{t}, A_{t+1}\right) \in \mathcal{A}_{t}$ and let $r:=W_{t}\left(z_{t}, A_{t+1}\right)$. For any $\hat{r} \in(0, \infty)$, let $\lambda:=\frac{\hat{r}}{r}$. Richness implies that there exists $\left(\hat{z}_{t}, A_{t+1}\right) \in \mathcal{A}_{t}$ such that

$$
\frac{P_{t}\left[\left(\hat{z}_{t}, A_{t+1}\right) \mid\left\{\left(z_{t}, A_{t+1}\right),\left(\hat{z}_{t}, A_{t+1}\right)\right\}\right]}{P_{t}\left[\left(z_{t}, A_{t+1}\right) \mid\left\{\left(z_{t}, A_{t+1}\right),\left(\hat{z}_{t}, A_{t+1}\right)\right\}\right]}=\lambda .
$$

By Lemma 4,

$$
\frac{\hat{r}}{r}=\frac{W_{t}\left(\hat{z}_{t}, A_{t+1}\right)}{W_{t}\left(z_{t}, A_{t+1}\right)}=\frac{W_{t}\left(\hat{z}_{t}, A_{t+1}\right)}{r}
$$

so $W_{t}\left(\hat{z}_{t}, A_{t+1}\right)=\hat{r}$. Since $\hat{r}$ was chosen arbitrarily, the conclusion follows.

\section{A.4. Proof of Lemma 5}

The necessity of Recursivity is immediate. For sufficiency, note that by Lemma 4, $\left\{P_{t}\right\}$ has a sequential Luce representation. Fix a period $t$ and an integer $n$. Let $\mathcal{M}_{t+1}^{n}$ be the subset of all menus from $\mathcal{M}_{t+1}$ with cardinality $n$. Recursivity together with the sequential Luce representation imply that $\succsim_{t}$ restricted to $\mathcal{M}_{t+1}^{n}$ is represented by $A_{t+1} \mapsto \sum_{a_{t+1} \in A_{t+1}} W_{t+1}\left(a_{t+1}\right)$. From the definitions of $\succsim_{t}$ and the sequential Luce representation, it follows that $\succsim_{t}$ restricted to $\mathcal{M}_{t+1}^{n}$ is also represented by the function $h_{t}$ restricted to $\mathcal{M}_{t+1}^{n}$. Thus, there exists a strictly increasing function $f_{t}^{n}$ such that, for all $A_{t+1} \in \mathcal{M}_{t+1}^{n}$, we have $h_{t}\left(A_{t+1}\right)=f_{t}^{n}\left(\sum_{a_{t+1} \in A_{t+1}} W_{t+1}\left(a_{t+1}\right)\right)$. Thus, for all $A_{t+1} \in \mathcal{M}_{t+1}$,

$$
h_{t}\left(A_{t+1}\right)=f_{t}^{\left|A_{t+1}\right|}\left(\sum_{a_{t+1} \in A_{t+1}} W_{t+1}\left(a_{t+1}\right)\right) .
$$

\section{A.5. Proof of Theorem 1}

The necessity of the axioms is straightforward. To prove sufficiency, we will use the following lemma. The solution to this functional equation is a classic result on $\mathbb{R}_{+}$, but we only have it defined on $\mathbb{N}$, so we give the proof.

LEMMA 6: $A$ monotone function $f: \mathbb{N} \rightarrow \mathbb{R}$ satisfies $\frac{f(l n)}{f(l m)}=\frac{f(n)}{f(m)}$ for all $l, m, n \in \mathbb{N}$ if and only if there exist $\beta>0$ and $\gamma \in \mathbb{R}$ such that $f(n)=\beta n^{\gamma}$.

Proof: Assume w.l.o.g. that $f$ is increasing. Set $m=1$ to get $f(\ln )=$ $f(l) f(n) / f(1)$. Define $g(t)=f(t) / f(1)$ for all $t \in \mathbb{N}$. Then $g(\ln )=$ $f(\ln ) / f(1)=f(l) f(n) /(f(1))^{2}=g(l) g(n)$, and $g\left(n^{k}\right)=(g(n))^{k}$ for all $n, k \in \mathbb{N}$. Fix $n \in \mathbb{N}$; for all rational $r=\frac{m}{k}<\frac{\log n}{\log 2}$, we have $(g(2))^{m}<(g(n))^{k}$, so 
$g(n)>(g(2))^{r}$. Take the supremum of such $r$ to obtain $g(n) \geq(g(2))^{\log n / \log 2}$. Likewise, considering $r$ larger than $\frac{\log n}{\log 2}$ and taking the infimum, we obtain $g(n)=(g(2))^{\log n / \log 2}=n^{\gamma}$ for some $\gamma \in \mathbb{R}$. By setting $\beta:=f(1)$, we get the desired conclusion.

Q.E.D.

STEP 1: Let $A_{t+1}, B_{t+1} \in \mathcal{M}_{t+1}, x, y \in Z$, and $n$ be an integer. Let $r:=$ $\sum_{a_{t+1} \in A_{t+1}} W_{t+1}\left(a_{t+1}\right)$ and $r^{\prime}:=\sum_{b_{t+1} \in B_{t+1}} W_{t+1}\left(b_{t+1}\right)$. Let $k:=\left|A_{t+1}\right|$ and $l:=$ $\left|B_{t+1}\right|$. Let $v:=v_{t}(x)$ and $v^{\prime}:=v_{t}(y)$. Lemma 5 and replica invariance imply that $P$ has a recursive adjusted Luce representation where the functions $\left\{G_{t}\right\}$ and $f_{t}^{n}$ satisfy

$$
\frac{G_{t}\left(v, f^{k}(r)\right)}{G_{t}\left(v, f^{l}\left(r^{\prime}\right)\right)}=\frac{G_{t}\left(v^{\prime n k}(n r)\right)}{G_{t}\left(v^{\prime n l}\left(n r^{\prime}\right)\right)} .
$$

Define $g_{t}^{n}(v, r):=G_{t}\left(v, f^{n}(n r)\right)$. The equation above implies

$$
\frac{g_{t}^{k}(v, r)}{g_{t}^{l}\left(v, r^{\prime}\right)}=\frac{g_{t}^{n k}\left(v^{\prime}, r\right)}{g_{t}^{n l}\left(v^{\prime}, r^{\prime}\right)}
$$

Equation (12) holds for all integers $n, k, l$, all $v, v^{\prime} \in R v_{t}$ (where $R v_{t}$ is the range of the function $v_{t}$ ) and all $r, r^{\prime} \in(0, \infty)$ (as Lemma 4 shows, Richness implies that the range of $W_{t+1}$ is $\left.(0, \infty)\right)$.

STEP 2: Fix $v=v^{\prime}$ and $r^{\prime}=r$, and define $\bar{g}_{t}(n):=g_{t}^{n}(v, r)$. Equation (12) implies that

$$
\frac{\bar{g}_{t}(n k)}{\bar{g}_{t}(n l)}=\frac{\bar{g}_{t}(k)}{\bar{g}_{t}(l)} .
$$

By Lemma 6, there exist $\beta_{t}>0$ and $\gamma_{t} \in \mathbb{R}$ such that $\bar{g}_{t}(n)=\beta_{t} n^{\gamma_{t}}$. Thus, there exist functions $\beta_{t}: R v_{t} \times(0, \infty) \rightarrow(0, \infty)$ and $\gamma_{t}: R v_{t} \times(0, \infty) \rightarrow \mathbb{R}$ such that

$$
g_{t}^{n}(v, r)=\beta_{t}(v, r) n^{\gamma_{t}(v, r)} .
$$

STEP 3: Fix $v=v^{\prime}$. Equations (12) and (13) imply that, for all $r, r^{\prime} \in(0, \infty)$,

$$
\frac{\beta_{t}(v, r) k^{\gamma_{t}(v, r)}}{\beta_{t}\left(v, r^{\prime}\right) l^{\gamma_{t}\left(v, r^{\prime}\right)}}=\frac{\beta_{t}(v, r)(n k)^{\gamma_{t}(v, r)}}{\beta_{t}\left(v, r^{\prime}\right)(n l)^{\gamma_{t}\left(v, r^{\prime}\right)}},
$$

which implies that $\gamma_{t}(v, r)=\gamma_{t}\left(v, r^{\prime}\right)$ for all $r, r^{\prime}$. Let $\gamma_{t}(v)$ denote this common value. Thus,

$$
g_{t}^{n}(v, r)=\beta_{t}(v, r) n^{\gamma_{t}(v)} .
$$


STEP 4: Fix $r, r^{\prime}, \lambda \in(0, \infty)$ and let $a_{t+1}, b_{t+1}, c_{t+1}, d_{t+1} \in M_{t+1}$ be such that $W_{t+1}\left(a_{t+1}\right)=r, W_{t+1}\left(b_{t+1}\right)=\lambda r, W_{t+1}\left(c_{t+1}\right)=r^{\prime}$, and $W_{t+1}\left(d_{t+1}\right)=\lambda r^{\prime}$, so that $P_{t+1}\left[a_{t+1} \mid\left\{a_{t+1}, b_{t+1}\right\}\right]=P_{t+1}\left[c_{t+1} \mid\left\{c_{t+1}, d_{t+1}\right\}\right]$. Probability Equivalence implies that, for any $x, y \in Z$,

$$
\begin{aligned}
P_{t} & {\left[\left(x,\left\{a_{t+1}\right\}\right) \mid\left\{\left(x,\left\{a_{t+1}\right\}\right),\left(x,\left\{b_{t+1}\right\}\right)\right\}\right] } \\
& =P_{t}\left[\left(y,\left\{c_{t+1}\right\}\right) \mid\left\{\left(y,\left\{c_{t+1}\right\}\right),\left(y,\left\{d_{t+1}\right\}\right)\right\}\right] .
\end{aligned}
$$

Thus, for all $v, v^{\prime} \in R v_{t}$ and all $r, r^{\prime}, \lambda \in(0, \infty)$, we have

$$
\frac{g_{t}^{1}(v, \lambda r)}{g_{t}^{1}(v, r)}=\frac{g_{t}^{1}\left(v^{\prime}, \lambda r^{\prime}\right)}{g_{t}^{1}\left(v^{\prime}, r^{\prime}\right)}
$$

This and equation (14) imply that

$$
\frac{\beta_{t}(v, \lambda r)}{\beta_{t}(v, r)}=\frac{\beta_{t}\left(v^{\prime}, \lambda r^{\prime}\right)}{\beta_{t}\left(v^{\prime}, r^{\prime}\right)}
$$

Let $v=v^{\prime}$ and $r^{\prime}=1$, and define $b_{t, v}(r):=\beta_{t}(v, r) / \beta_{t}(v, 1)$. The equation above implies the Cauchy functional equation $b_{t, v}(\lambda r)=b_{t, v}(\lambda) b_{t, v}(r)$. Since the function $g_{t}^{1}(v, \cdot)$ is increasing, the function $\beta_{t}(v, \cdot)$ is increasing, so the function $b_{t, v}(\cdot)$ is increasing and hence continuous at some point. By Theorem 3, Section 2.1 of Aczél (1966), the only nonzero solutions of this equation are of the form $b_{t, v}(r)=r^{\delta_{t}(v)}$ for some $\delta_{t}(v)>0$. Thus, equation (15) implies that $\lambda^{\delta_{t}(v)}=\lambda^{\delta_{t}\left(v^{\prime}\right)}$, and since $\lambda$ is arbitrary, we have $\delta_{t}(v)=\delta_{t}\left(v^{\prime}\right)$ for all $v, v^{\prime} \in(0, \infty)$. Let $\delta_{t}>0$ denote their common value. Thus, $b_{t, v}(r)=r^{\delta_{t}}$; this implies that $\beta_{t}(v, r)=\beta_{t}(v, 1) r^{\delta_{t}}$. Define $B(v):=\beta_{t}(v, 1)$. We have proven that

$$
g_{t}^{n}(v, r)=B_{t}(v) r^{\delta_{t}} n^{\gamma_{t}(v)}
$$

STEP 5: Equations (12) and (16) with $k=2, l=1$ imply that $2^{\gamma_{t}(v)}=2^{\gamma_{t}\left(v^{\prime}\right)}$, which implies that $\gamma_{t}(v)=\gamma_{t}\left(v^{\prime}\right)$ for all $v, v^{\prime} \in(0, \infty)$. Let $\gamma_{t}$ denote the common value. We have proven that

$$
g_{t}^{n}(v, r)=B_{t}(v) r^{\delta_{t}} n^{\gamma_{t}}
$$

STEP 6: By definition in Step 1, equation (17) implies that $G_{t}\left(v, f^{n}(r)\right)=$ $g_{t}^{n}(v, r / n)=B_{t}(v) r^{\delta_{t}} n^{\gamma_{t}-1}$. Define $\hat{v}_{t}(z):=\log B_{t}\left(v_{t}(z)\right)$ and $\kappa_{t}:=-\left(\gamma_{t}-1\right) / \delta_{t}$. We have

$$
\log W_{t}\left(z_{t}, A_{t+1}\right)=\hat{v}_{t}\left(z_{t}\right)+\delta_{t} \log \left(\sum_{a_{t+1} \in A_{t+1}} W_{t+1}\left(a_{t+1}\right)\right)-\delta_{t} \kappa_{t} \log \left|A_{t+1}\right| .
$$


STEP 7: We claim next that the functions $\hat{v}_{t}$ are surjective. To see this, fix $A_{t+1} \in \mathcal{M}_{t+1}$ and let $d:=\delta_{t} \sum_{a_{t+1} \in A_{t+1}} W_{t+1}\left(a_{t+1}\right)-\delta_{t} \kappa_{t} \log \left|A_{t+1}\right|$. Lemma 4 and (18) imply that, for any $r \in(0, \infty)$, there exists $z \in Z$ such that $r=$ $W_{t}\left(z, A_{t+1}\right)=\exp \left(\hat{v}_{t}\left(z_{t}\right)+d\right)$. Thus, for any $r^{\prime} \in \mathbb{R}$, there exists $z \in Z$ such that $\hat{v}_{t}(z)=r^{\prime}$ by letting $r=\exp \left(r^{\prime}+d\right)$. by

STEP 8: Given (18), the preference over consumption streams is represented

$$
\tilde{z} \mapsto \sum_{t=0}^{T}\left(\prod_{s=0}^{t-1} \delta_{s}\right) \hat{v}_{t}\left(z_{t}\right) .
$$

Let $v:=\hat{v}_{0}$ and $\delta:=\delta_{0}$. From the proof of Theorem 7.5 of Fishburn (1970), ${ }^{19}$ it follows that if Stream Stationarity is satisfied, then $\hat{v}_{t} \equiv v$ for all $t$ and $\delta_{t}=\delta$ for all $t$.

STEP 9: Given (18), and the stationarity of $v$ and $\delta$, the induced preference on consumption streams is represented by $\tilde{z} \mapsto \sum_{t=0}^{T} \delta^{t} v\left(z_{t}\right)$, with $(z, \ldots, z) \succ_{0}$ $\left(z^{\prime}, \ldots, z^{\prime}\right)$ if and only if $v(z)>v\left(z^{\prime}\right)$ and $\left(z_{0}, \ldots, z_{t-1}, z, z^{\prime}, z_{t+2}, \ldots, z_{T}\right) \succsim_{0}$ $\left(z_{0}, \ldots, z_{t-1}, z^{\prime}, z, z_{t+2}, \ldots, z_{T}\right)$ if and only if $v(z)+\delta v\left(z^{\prime}\right)>v\left(z^{\prime}\right)+\delta v(z)$. Thus, by Impatience, $\left(v(z)-v\left(z^{\prime}\right)\right)(1-\delta)>0$, which implies that $\delta<1$.

STEP 10: Let $A_{1} \in \mathcal{M}_{1}$ and $A_{t+1} \in \mathcal{M}_{t+1}$ for some $t<T$. Let $d_{1}:=$ $\sum_{a_{1} \in A_{1}} W_{1}\left(a_{1}\right), n_{1}:=\left|A_{1}\right|, d_{t+1}:=\sum_{a_{t+1} \in A_{t+1}} W_{t+1}\left(a_{t+1}\right)$, and $n_{t+1}:=\left|A_{t+1}\right|$. Stationary Choice Aversion implies that

$$
\frac{d_{1}^{\delta} n_{1}^{-\delta \kappa_{0}}}{d_{1}^{\delta} n_{1}^{-\delta \kappa_{0}}+\left(2 d_{1}\right)^{\delta}\left(2 n_{1}\right)^{-\delta \kappa_{0}}}=\frac{d_{t+1}^{\delta} n_{t+1}^{-\delta \kappa_{t}}}{d_{t+1}^{\delta} n_{t+1}^{-\delta \kappa_{t}}+\left(2 d_{t+1}\right)^{\delta}\left(2 n_{t+1}\right)^{-\delta \kappa_{t}}},
$$

which implies that $\kappa_{0}=\kappa_{t}$.

\section{A.6. Proofs of Other Results}

\section{A.6.1. Proof of Proposition 2}

To see why this is true, suppose that $A_{t+1} \succsim_{t}^{*}\left(\succ_{t}^{*}\right) B_{t+1}$. Let $m_{A}:=$ $\max _{q \in \Delta(A)} \sum q\left(a_{t+1}\right) U_{t+1}\left(a_{t+1}\right)+H^{|A|}(q)$ and $m_{B}=\max _{q \in \Delta(B)} \sum q\left(a_{t+1}\right) \times$ $U_{t+1}\left(a_{t+1}\right)+H_{\kappa^{*}}^{|B|}(q)$. Then

$$
m_{A}-\kappa^{*} \log |A| \geq(>) m_{B}-\kappa^{*} \log |B|
$$

${ }^{19}$ Our induced preference on consumption streams may not satisfy the continuity property that Fishburn requires. However, the cardinal uniqueness of additive representations invoked in his proof holds due to the surjectivity of the $\hat{v}_{t}$ functions. 
iff

$$
\kappa^{*}(\log |B|-\log |A|) \geq(>) m_{B}-m_{A} .
$$

The above inequality holds if we replace $\kappa^{*}$ with $\kappa$ as long as $\kappa \geq \kappa^{*}$.

To prove the converse, suppose that $\kappa<\kappa^{*}$. By Richness, there exist $A_{t+1}=$ $\left\{a_{t+1}\right\}$ and $B_{t+1}=\left\{b_{t+1}, b_{t+1}^{\prime}\right\}$ such that $m_{A}=m_{B}-\kappa^{*} \log 2$, so that $A_{t+1} \succsim^{*} B_{t+1}$. If $P$ were more choice averse than $P^{*}$, we would have $A_{t+1} \succsim B_{t+1}$. But $m_{A}<$ $m_{B}-\kappa \log 2$, so $A_{t+1} \prec B_{t+1}$, a contradiction.

\section{A.6.2. Proof of Proposition 3}

Let $n=\left|A_{t+1}\right|$ and note that $A_{t+1} \cup\left\{b_{t+1}\right\} \succ_{t} A_{t+1}$ whenever

$$
\begin{gathered}
\log \left(e^{U\left(b_{t+1}\right)}+\sum_{a_{t+1} \in A_{t+1}} e^{U\left(a_{t+1}\right)}\right)-\kappa \log (n+1) \\
>\log \left(\sum_{a_{t+1} \in A_{t+1}} e^{U\left(a_{t+1}\right)}\right)-\kappa \log n
\end{gathered}
$$

iff

$$
\frac{1}{(n+1)^{\kappa}}\left[e^{U\left(b_{t+1}\right)}+\sum_{a_{t+1} \in A_{t+1}} e^{U\left(a_{t+1}\right)}\right]>\frac{1}{n^{\kappa}} \sum_{a_{t+1} \in A_{t+1}} e^{U\left(a_{t+1}\right)}
$$

iff

$$
\frac{\sum_{a_{t+1} \in A_{t+1}} e^{U\left(a_{t+1}\right)}}{e^{U\left(b_{t+1}\right)}+\sum_{a_{t+1} \in A_{t+1}} e^{U\left(a_{t+1}\right)}}<\frac{n^{\kappa}}{(n+1)^{\kappa}}
$$

iff

$$
P_{t+1}\left[b_{t+1} \mid A_{t+1} \cup\left\{b_{t+1}\right\}\right]>1-\frac{n^{\kappa}}{(n+1)^{\kappa}} .
$$

\section{A.6.3. Proof of Proposition 4}

For the proof, we will use the DALu representation, noting that $A_{t+1} \succsim_{t} B_{t+1}$ iff

$$
\left|B_{t+1}\right|^{\kappa} \sum_{a_{t+1} \in A_{t+1}} W_{t+1}\left(a_{t+1}\right) \geq\left|A_{t+1}\right|^{\kappa} \sum_{b_{t+1} \in B_{t+1}} W_{t+1}\left(b_{t+1}\right),
$$

and that all of the weights $W_{t+1}$ are positive. 
PART 1: Suppose that $\kappa \geq 1$ and $A_{t+1}$ and $B_{t+1}$ are disjoint. Then $\left|A_{t+1} \cup B_{t+1}\right|^{\kappa} \geq\left|A_{t+1}\right|^{\kappa}+\left|B_{t+1}\right|^{\kappa}$ since the function $x \mapsto x^{\kappa}$ is superadditive (see proof of Proposition 5). Suppose that $A_{t+1} \succsim_{t} B_{t+1}$ and add $\left|A_{t+1}\right|^{\kappa} \sum_{a_{t+1} \in A_{t+1}} W_{t+1}\left(a_{t+1}\right)$ to each side of $(*)$ to obtain $\left(\left|A_{t+1}\right|^{\kappa}+\left|B_{t+1}\right|^{\kappa}\right) \times$ $\sum_{a_{t+1} \in A_{t+1}} W_{t+1}\left(a_{t+1}\right) \geq\left|A_{t+1}\right|^{\kappa} \sum_{a_{t+1} \in A_{t+1} \cup B_{t+1}} W_{t+1}\left(a_{t+1}\right)$. Thus $A_{t+1} \succsim_{t} B_{t+1}$ implies $A_{t+1} \succsim_{t} A_{t+1} \cup B_{t+1}$. Conversely, suppose $\kappa<1$. By Richness, there is a pair of actions $a_{t+1}, b_{t+1}$ with $\frac{2^{\kappa}-1}{2^{\kappa}}<P_{t+1}\left[b_{t+1} \mid\left\{a_{t+1}, b_{t+1}\right\}\right]<\frac{1}{2}$, so that $\left\{a_{t+1}\right\} \succsim_{t}\left\{b_{t+1}\right\}$. Then by Proposition $3,\left\{a_{t+1}, b_{t+1}\right\} \succ_{t}\left\{a_{t+1}\right\}$, which contradicts positive set betweenness.

PART 2: Suppose that $\kappa \leq 1$ and $A_{t+1}$ and $B_{t+1}$ are disjoint. We have $\mid A_{t+1} \cup$ $\left.B_{t+1}\right|^{\kappa} \leq\left|A_{t+1}\right|^{\kappa}+\left|B_{t+1}\right|^{\kappa}$ since the function $x \mapsto x^{\kappa}$ is subadditive (see proof of Proposition 5). $A_{t+1} \succsim_{t} B_{t+1}$ and add $\left|B_{t+1}\right|^{\kappa} \sum_{b_{t+1} \in B_{t+1}} W_{t+1}\left(b_{t+1}\right)$ to each side of $(*)$ to obtain $\left|B_{t+1}\right|^{\kappa} \sum_{a_{t+1} \in A_{t+1} \cup B_{t+1}} W_{t+1}\left(a_{t+1}\right) \geq\left(\left|A_{t+1}\right|^{\kappa}+\left|B_{t+1}\right|^{\kappa}\right) \times$ $\sum_{b_{t+1} \in B_{t+1}} W_{t+1}\left(b_{t+1}\right)$. Thus, $A_{t+1} \succsim_{t} B_{t+1}$ implies $A_{t+1} \cup B_{t+1} \succsim_{t} B_{t+1}$. Conversely, suppose $\kappa>1$. By Richness, there exist actions $a_{t+1}, b_{t+1}$ with $\frac{1}{2}<P_{t+1}\left[a_{t+1} \mid\left\{a_{t+1}, b_{t+1}\right\}\right]<\frac{2^{\kappa}-1}{2^{\kappa}}$ so that $\left\{a_{t+1}\right\} \succsim_{t}\left\{b_{t+1}\right\}$, and by Proposition 3, $\left\{b_{t+1}\right\} \succ_{t}\left\{a_{t+1}, b_{t+1}\right\}$, which contradicts negative set betweenness.

\section{PART 3: Follows from Parts 1 and 2.}

PARTS 4 AND 5: Suppose that $\kappa \leq 0$. If $A_{t+1} \supseteq B_{t+1}$, then $\left|A_{t+1}\right|^{-\kappa} \geq\left|B_{t+1}\right|^{-\kappa}$; therefore, $\left|A_{t+1}\right|^{-\kappa} \sum_{a_{t+1} \in A_{t+1}} W_{t+1}\left(a_{t+1}\right) \geq\left|B_{t+1}\right|^{-\kappa} \sum_{a_{t+1} \in B_{t+1}} W_{t+1}\left(a_{t+1}\right)$, so $A_{t+1} \succsim_{t} B_{t+1}$. Conversely, suppose that $\kappa>0$ and fix an arbitrary $A_{t+1}$. Since Richness implies that the range of the function $W_{t+1}$ is $(0,+\infty)$, it follows that there exists $b_{t+1}$ with $P_{t+1}\left[b_{t+1} \mid A_{t+1} \cup\left\{b_{t+1}\right\}\right]<\frac{2^{\kappa}-1}{2^{\kappa}}$, and from Proposition 3 it follows that $A_{t+1} \succ_{t} A_{t+1} \cup\left\{b_{t+1}\right\}$.

\section{A.6.4. Proof of Proposition 5}

To see this formally, let $F(T):=\sum_{t=1}^{T-1} \delta^{t-1} v\left(z_{t}\right)$ be the value of intermediate consumption and note that the value of choosing early is $v\left(z_{0}\right)+$ $\delta \log \left(\sum_{\tilde{z}_{T} \in A} \exp \left(F(T)+\delta^{T-1} v\left(\tilde{z}_{T}\right)\right)\right)-\kappa \log |A|$, whereas the value of choosing late is $v\left(z_{0}\right)+\delta\left(F(T)+\delta^{T-1} \log \left(\sum_{\tilde{z}_{T} \in A} \exp v\left(\tilde{z}_{T}\right)\right)-\delta^{T-1} \kappa \log |A|\right)$. Thus,

$$
\begin{aligned}
r= & \frac{P_{0}\left[a_{0} \mid\left\{a_{0}, b_{0}\right\}\right]}{P_{0}\left[b_{0} \mid\left\{a_{0}, b_{0}\right\}\right]} \\
= & \exp \left(v\left(z_{0}\right)+\delta \log \left(\sum_{\tilde{z}_{T} \in A} \exp \left(F(T)+\delta^{T-1} v\left(\tilde{z}_{T}\right)\right)\right)-\delta \kappa \log |A|\right) \\
& / \exp \left(v\left(z_{0}\right)+\delta(F(T)\right. \\
& \left.\left.+\delta^{T-1} \log \left(\sum_{\tilde{z}_{T} \in A} \exp v\left(\tilde{z}_{T}\right)\right)-\delta^{T-1} \kappa \log |A|\right)\right)
\end{aligned}
$$




$$
=\left[\frac{\sum_{\tilde{z}_{T} \in A} \exp \left(v\left(\tilde{z}_{T}\right)\right)^{\delta^{T-1}}}{\left(\sum_{\tilde{z}_{T} \in A} \exp v\left(\tilde{z}_{T}\right)\right)^{\delta^{T-1}}} e^{\left(\delta^{T-1}-1\right) \kappa \log |A|}\right]^{\delta} .
$$

To show part $(1)$, note that $e^{\left(\delta^{T-1}-1\right) \log |A|}<1$. Thus, a decrease in $\kappa$ increases $r$.

To show part (2), note that when $\kappa=0, r>1$ follows from the facts that $|A| \geq 2$, that with $\delta<1$ the function $z \mapsto z^{\delta^{T-1}}$ is strictly concave, and that strictly concave functions that pass through zero are strictly subadditive. ${ }^{20}$ The result for $\kappa<0$ follows from part (1). When $\kappa=1, r \leq 1$ by Jensen's inequality since $\delta<1$ and the function $z \mapsto z^{\delta^{T-1}}$ is strictly concave. The equality holds if and only if all the elements of $A$ are equivalents. For $\kappa>1, r<1$ by part (1).

\section{REFERENCES}

ACKerberG, D. A., AND M. RYSMAN (2005): "Unobservable Product Differentiation in Discrete Choice Models: Estimating Price Elasticities and Welfare Effects," RAND Journal of Economics, 36, 771-788. [658]

ACZÉL, J. (1966): Lectures on Functional Equations and Their Applications. New York: Academic Press. [685]

Aguirregabiria, V., AND P. Mira (2010): “Dynamic Discrete Choice Structural Models: A Survey," Journal of Econometrics, 156 (1), 38-67. [652,656]

AHN, D. S., AND T. SARVER (2013): "Preference for Flexibility and Random Choice," Econometrica, 81 (1), 341-361. [657,676]

Anderson, S. P., A. De Palma, And J. F. Thisse (1992): Discrete Choice Theory of Product Differentiation. Cambridge, MA: MIT Press. [652,678]

BARBERÁ, S., AND P. K. PATTANAIK (1986): "Falmagne and the Rationalizability of Stochastic Choices in Terms of Random Orderings," Econometrica, 53, 707-715. [676]

BERRY, S., J. LEVINSOHN, AND A. PAKES (1995): "Automobile Prices in Market Equilibrium," Econometrica, 63, 841-890. [676]

DeKel, E., AND B. L. LIPMAN (2012): “Costly Self-Control and Random Self-Indulgence," Econometrica, 80, 1271-1302. [677]

DEKEL, E., B. L. LIPMAN, AND A. RUSTICHINI (2001): "Representing Preferences With a Unique Subjective State Space," Econometrica, 69 (4), 891-934. [652,665] [665]

Dillenberger, D., J. S. Lleras, P. SADOwski, AND N. TAKeOKA (2013): “A Theory of Subjective Learning," Report. [657]

ECKSTEIN, Z., AND K. I. WOLPIN (1989): "The Specification and Estimation of Dynamic Stochastic Discrete Choice Models: A Survey,” Journal of Human Resources, 24 (4), 562-598. [652]

\footnotetext{
${ }^{20}$ This is a standard result; we include a proof here for completeness: Suppose $f$ is strictly concave and $f(0)=0$. Then for $t \in(0,1), f(t x)=f(t x+(1-t) 0)>t f(x)+(1-t) 0=t f(x)$. Hence, $f(x)+f(y)=f\left((x+y) \frac{x}{(x+y)}\right)+f\left((x+y) \frac{y}{(x+y)}\right)>\left(\frac{x}{x+y}\right) f(x+y)+\left(\frac{y}{x+y}\right) f(x+y)=$ $f(x+y)$.
} 
EPSTEIN, L. G., AND S. E. ZIN (1989): "Substitution, Risk Aversion, and the Temporal Behavior of Consumption and Asset Returns: A Theoretical Framework," Econometrica, 57, 937-969. [669]

ERgin, H. (2003): “Costly Contemplation,” Report. [676]

ERgIN, H., AND T. SARVER (2010): "A Unique Costly Contemplation Representation," Econometrica, 78 (4), 1285-1339. [676]

FALMAGNE, J. C. (1978): “A Representation Theorem for Finite Random Scale Systems," Journal of Mathematical Psychology, 18 (1), 52-72. [676]

FisHBURN, P. C. (1970): Utility Theory for Decision Making. New York: Wiley. [671,674,686]

FudENBERG, D., AND D. K. LEVINE (1995): “Consistency and Cautious Fictitious Play,” Journal of Economic Dynamics and Control, 19, 1065-1089. [677]

(2006): "A Dual-Self Model of Impulse Control," American Economic Review, 96, 1449-1476. [677]

(2011): "Risk, Delay, and Convex Self-Control Costs," American Economic Journal: Microeconomics, 3 (3), 34-68. [677]

- (2012): "Timing and Self-Control," Econometrica, 80 (1), 1-42. [677]

FUDENBERG, D., AND S. TAKAHASHI (2011): "Heterogeneous Beliefs and Local Information in Stochastic Fictitious Play," Games and Economic Behavior, 71, 100-120. [677]

FudENBERG, D., R. IIJIMA, AND T. STRZALECKI (2014): "Stochastic Choice and Revealed Perturbed Utility," Report. [653,658,676]

GowrisankaRAN, G., AND M. RYSMAN (2012): "Dynamics of Consumer Demand for New Durable Goods," Journal of Political Economy, 120, 1173-1219. [652,662]

Gul, F., AND W. PESENDORFER (2001): "Temptation and Self-Control," Econometrica, 69 (6), 1403-1435. [665]

(2006): "Random Expected Utility," Econometrica, 74 (1), 121-146. [676]

Gul, F., P. NATENZON, AND W. PESENDORFER (2014): "Random Choice as Behavioral Optimization," Econometrica, 82 (5), 1873-1912. [676]

Hannan, J. (1957): "Approximation to Bayes Risk in Repeated Plays," in Contributions to the Theory of Games, Vol. 3, ed. by M. Dresher, A. Tucker, and P. Wolfe. Princeton, NJ: Princeton University Press, 97-139. [677]

HARSANYI, J. C. (1973a): "Games With Randomly Disturbed Payoffs: A New Rationale for Mixed-Strategy Equilibrium Points," International Journal of Game Theory, 2 (1), 1-23. [653, 675]

(1973b): "Oddness of the Number of Equilibrium Points: A New Proof," International Journal of Game Theory, 2 (1), 235-250. [653]

HART, S., AND A. MAS-COLELL (2001): "A General Class of Adaptive Strategies," Journal of Economic Theory, 98 (1), 26-54. [677]

HENDEL, I., AND A. NEVO (2006): "Measuring the Implications of Sales and Consumer Inventory Behavior," Econometrica, 74 (6), 1637-1673. [652,657]

HofBauer, J., AND E. Hopkins (2005): "Learning in Perturbed Asymmetric Games," Games and Economic Behavior, 52 (1), 133-152. [677]

HofBaUer, J., AND W. H. SANDHOLM (2002): "On the Global Convergence of Stochastic Fictitious Play," Econometrica, 70 (6), 2265-2294. [677]

JACKSON, M., AND L. YARIV (2010): "Collective Dynamic Choice: The Necessity of Time Inconsistency," Report. [676]

KENNAN, J., AND J. R. WALKER (2011): "The Effect of Expected Income on Individual Migration Decisions," Econometrica, 79 (1), 211-251. [652]

Koopmans, T. C. (1960): "Stationary Ordinal Utility and Impatience," Econometrica, 28 (2), 287-309. [654,670,671,673,675,676,680,682]

KREPS, D. M. (1979): "A Representation Theorem for 'Preference for Flexibility',” Econometrica, 47, 565-577. [652,665,675,677]

KREPS, D. M., AND E. L. PORTEUS (1978): "Temporal Resolution of Uncertainty and Dynamic Choice Theory,” Econometrica, 46, 185-200. [669,672,680] 
Krishna, R. V., AND P. SAdOwSKI (2014): “Dynamic Preference for Flexibility,” Econometrica, $82(2), 655-703 .[677]$

LU, J. (2013): "Random Choice and Private Information," Unpublished Manuscript, Princeton University. [657]

LUCE, D. (1959): Individual Choice Behavior. New York: Wiley. [654,670,675,679]

MANZINI, P., AND M. MARIOTTI (2014): "Stochastic Choice and Consideration Sets," Econometrica, 82, 1153-1176. [653]

MARSCHAK, J. (1959): “Binary Choice Constraints on Random Utility Indicators,” Discussion Paper 74, Cowles Foundation for Research in Economics, Yale University. [653,675]

MatTsSon, L.-G., AND J. W. WeIBull (2002): "Probabilistic Choice and Procedurally Bounded Rationality," Games and Economic Behavior, 41, 61-78. [653]

MCFADDEN, D. (1973): "Conditional Logit Analysis of Qualitative Choice Behavior," in Frontiers in Econometrics, ed. by P. Zarembka. New York: Academic Press, 105-142. [653,670]

MiLleR, R. A. (1984): "Job Matching and Occupational Choice," Journal of Political Economy, 92, 1086-1120. [652]

Montiel Olea, J. L., AND T. Strzalecki (2014): "A Simple Axiomatization of QuasiHyperbolic Discounting," Quarterly Journal of Economics, 129, 1449-1499. [676]

NOOR, J., AND N. TAKEOKA (2010a): "Menu-Dependent Self-Control," Report. [677]

- (2010b): "Uphill Self-Control," Theoretical Economics, 5 (2), 127-158. [677]

Ortoleva, P. (2013): “The Price of Flexibility: Towards a Theory of Thinking Aversion,” Journal of Economic Theory, 148 (3), 903-934. [676]

Rubinstein, A., AND Y. SAlAnt (2006): "A Model of Choice From Lists," Theoretical Economics, 1, 3-17. [669]

RuST, J. (1987): "Optimal Replacement of GMC Bus Engines: An Empirical Model of Harold Zurcher," Econometrica, 55, 999-1033. [651]

(1989): "A Dynamic Programming Model of Retirement Behavior," in The Economics of Aging, ed. by D. Wise. Chicago: University of Chicago Press, 359-398. [652]

(1994): "Structural Estimation of Markov Decision Processes," in Handbook of Econometrics, Vol. 4. Amsterdam: North-Holland, 3081-3143. [652,657]

Swait, J., AND A. A. J. MARLEY (2013): "Probabilistic Choice (Models) as a Result of Balancing Multiple Goals," Journal of Mathematical Psychology, 57, 1-14. [654]

Sweeting, A. (2011): "Dynamic Product Positioning in Differentiated Product Markets: The Effect of Fees for Musical Performance Rights on the Commercial Radio Industry,” Report. [652]

Thurstone, L. L. (1927): “A Law of Comparative Judgment,” Psychological Review, 34 (4), 273-286. [653]

Train, K. E. (2009): Discrete Choice Methods With Simulation (Second Ed.). Cambridge: Cambridge University Press. [659,660,678]

VAN DAMME, E. (1991): Stability and Perfection of Nash Equilibria. New York: Springer. [653]

VAN DAMme, E., AND J. W. WeIBull (2002): "Evolution in Games With Endogenous Mistake Probabilities," Journal of Economic Theory, 106 (2), 296-315. [653]

Dept. of Economics, Harvard University, Littauer Center, 1805 Cambridge Street, Cambridge, MA 02138, U.S.A.; dfudenberg@harvard.edu

and

Dept. of Economics, Harvard University, Littauer Center, 1805 Cambridge

Street, Cambridge, MA 02138, U.S.A.; tomasz_strzalecki@harvard.edu.

Manuscript received August, 2013; final revision received October, 2014. 